Discussion Paper No. 986

\title{
THE PROBLEM \\ OF MULTIPLE COMMONS: \\ A MARKET DESIGN APPROACH
}

\author{
Ryan Tierney
}

December 2016

The Institute of Social and Economic Research Osaka University

6-1 Mihogaoka, Ibaraki, Osaka 567-0047, Japan 


\title{
THE PROBLEM OF MULTIPLE COMMONS: A MARKET DESIGN APPROACH
}

\author{
RYAN TIERNEY \\ Université de Montréal \\ Pavillon Lionel-Groulx, CIREQ \\ CP 6128, succursale Centre-ville \\ Montréal QC H3C $3 \mathrm{J7}$
}

\begin{abstract}
Aвstract. There are several locations, each of which is endowed with a resource that is specific to that location. Examples include coastal fisheries, oil fields, etc. Each agent will go to a single location and harvest some of the resource there. Several agents may go to each location. We assign harvesting rights based on preferences alone, though we later extend the model to accommodate private endowments of money. We find the best allocation rule in the class of rules that are strategy-proof, anonymous, and that satisfy a weak continuity property. We also find an ascending mechanism, similar to an auction, that implements the rule. The rule coincides with a special simulated price equilibrium, wherein agents buy their desired resource with tokens distributed by the social planner. Equilibrium price vectors form a lower semi-lattice and thus there is a unique minimal price vector. The equilibria associated with the minimal price vector are called min-price Walrasian equilibria. These equilibria form an essentially single-valued correspondence, and this correspondence is the rule we characterize.
\end{abstract}

E-mail address: ryanetierney@gmail.com.

Date: December 15, 2016.

Key words and phrases. Incentive-compatibility, min-price equilibrium, commons, indivisible goods.

Many thanks to my advisor William Thomson. Thanks also to Tommy Andersson whose generous feedback improved exposition. Comments from Patrick Harless, Tomoya Kazumura, Andy MacKenzie, Thomas Gall, Agustin Bonifacio, Vikram Manjunath, Sean Horan, participants of the 2014 Social Choice and Welfare conference in Boston, and participants of the 2014 International Workshop on Game Theory in Sao Paulo are gratefully acknowledged. A precursor to this paper appears as Chapter 3 of the author's dissertation, completed, with financial support, at the University of Rochester. 


\section{INTRODUCTION}

The simplest solution to the problem of a commons (Hardin, 1968) is to eliminate it; divide the resource among the relevant agents. The division of a resource, however, is a non-trivial problem, especially as a commons is typically not uniform. In such a case, there is no obvious notion of equal splitting as it is not clear what is "equal." In many cases, though, there is a clear, finite partition of a commons into homogeneous parts. This paper studies how to assign each agent to a single such part and to how to decide the size of his allotment. We impose a weak equity requirement (anonymity in welfare terms) and dominant strategy incentive compatibility. Our main result is that, subject to a weak continuity condition, there is a unique constrained optimal, or second-best, rule satisfying these requirements. We identify this rule and a way to calculate it.

For an instance of the problem we address, consider fisheries. The coastal fisheries of the United States are divided into 9 regions, the authority over each being delegated to a council. The mandate of the councils includes, among other things, "ensuring the equitable allocation of fishing privileges, preventing excessive accumulation of quota, using fishery resources efficiently, . . ., and considering the importance of fishery resources to fishing communities" (GAO (2002)). One method for achieving this mandate, which has been used in Alaska, the Mid-Atlantic, and the South Atlantic, is the Individual Fishing Quota (IFQ) system. In Alaska, halibut and sablefish are regulated by IFQ's. The fishery of each species is divided into zones, 8 for halibut and 6 for sablefish. For each species and each of its zones, the council sets a maximum allowable catch, based on scientific assessment of the current health of the stock. The allowable catch is then distributed among eligible entities. This is a commons with 14 distinct parts, each effectively homogeneous. The same fish caught in different zones entails different costs, in terms of travel, labour, and danger. Thus, the fishers may have complex preferences over where they go, which fish they may catch, and how much.

We present further applications below, but let us first fix a theoretical framework. There is a finite population of agents and a finite number of sites. Each agent will go to a single site and consume some of the unique resource present there. ${ }^{1}$ Several agents may go to the same site, in which case the resource of that site must be divided among them. Agents have preferences over the

\footnotetext{
${ }^{1}$ The vast majority of fishers in the data (http://alaskasheries.noaa.gov/ram/ifqreports.htm) specialize in a single species, at least per-outing. A greater threat to the practicality of the model is that many fishers work in several zones; however, much of this is due to fragmentation caused by some zones with low quota. For halibut, for example, by constructing a coarser partition, with 4 zones, I found that $80 \%$ of fishing entities work in only one zone. Moreover, this is a very conservative figure, as it doesn't control for the fact that many entities operate several boats.
} 
two dimensions of site and quantity and these preferences are private information. The problem is to elicit preferences and allocate resources in the best way possible. Note that the "sites" and their resources need not be geographical locations and physical objects. For example, each site may be a computer or club membership, and the resource divided might be usage time or any other excludable privilege.

One simple solution is to impose an exogenous order on agents and allow each agent to take his desired bundle in turn. We confront a choice: which order should we use? Note that randomizing does not eliminate the seriousness of this choice. Since agents prefer more of each resource to less, each agent will, at his turn, take the entire endowment of a site. Thus, if there are more agents than sites, randomized serial dictatorship will leave some agents with nothing, ex post. To eliminate this extreme inequity, for which our benchmark model has no justification, we impose an anonymity condition. As is frequently seen in the literature (see section 1.1), no anonymous rule is Pareto efficient (henceforth simply efficient) and elicits true preferences as a weakly dominant strategy.

However, we show that there is a unique constrained efficient rule. More precisely, we show there is a most efficient rule in the set of rules satisfying dominant-strategy incentive compatibility, anonymity in welfare terms, and a weak continuity condition. We identify and characterize this rule, which we denote $F^{*}$. This rule fails to be efficient as it may leave some resources undistributed, but we find that $F^{*}$ approaches efficiency as the number of agents increases. Finally, we also find an auction-type mechanism for calculating $F^{*}$, meaning that it can easily be realized in practice.

While $F^{*}$ is an abstract mapping from states of the world to allocations, it happens to be the outcome of a certain type of simulated price equilibrium. A quantity of numeraire, having no real value, is distributed among agents. For each site, there is a unit price of its resource, in terms of numeraire. Each agent then "purchases" a quantity of resource at one of the sites. Many price vectors will yield feasible (not necessarily market-clearing) allocations. We show that there is a minimal such vector and the set of equilibria associated with this minimal price is $F^{*}$.

Anonymity implies that each agent is given the same quantity of numeraire; however, we show that the existence of the minimal price equilibrium does not require equal distribution of numeraire. Thus, we extend $F^{*}$ to a finite-dimensional family of rules, allowing for differential treatment of agents. In fact, we are able to extend the rule even further: we may assume that agents value consumption of the numeraire, in which case we simply call it money. 
Applications of these extensions include graduate program admissions and the leasing of oil field contracts. In some countries, admission to graduate schools depends largely on an exam score. Thus, we let each student's endowment of numeraire be his score. Each department is a site and its resource is its budget for stipends. The student uses his numeraire to "purchase" acceptance to a department, along with a fraction of the total stipends available there. In this case, the price for a given department provides the rate for converting numeraire into that department's stipend. For a country leasing oil contracts, each drilling unit has an endowment of money, and each site is an oil field. Demand is defined in the natural way, and, again, a minimal price equilibrium exists.

We introduce the formal model in Section 2. In Section 3 we introduce a simulated equilibrium that allows us to describe $F^{*}$. We analyse the properties of the rule and provide some comparative statics. Section 4 gives the characterization and section 5 the auction mechanism. Section 6 discards the anonymity requirement and enriches the model to include money.

1.1. Background and Contribution. We study a model in which each agent in society travels to one of several pools of resource and harvests some of the special resource there. Several agents may simultaneously harvest from the same pool. Such a problem has been studied before (Gensemer et al. (1998); Ackermann et al. (2007); Monderer and Shapley (1996); Gourvès et al. (2012) and others), but, to my knowledge, not when disposal of some resources is permitted, and not in the context of mechanism design.

To understand how the model here relates to what is known, consider the following special case: suppose that one of the resource pools admits harvesting by all agents, and that each of the other pools admits harvesting by at most one agent. The resulting model is isomorphic to a model of assigning objects and money: We say an agent consumes an "object" if he is assigned to a capacityconstrained resource, and otherwise he consumes the "null object." The "money" consumed is just the quantity of resource consumed, regardless of where. Since we admit arbitrary preferences, there is no restriction on the wealth effect, and thus money consumed together with object $a$ may be significantly different, in the eyes of the consumer, from money consumed together with object $b$. There remains, however, a significant difference between this special case of our model and the canonical model of objects-and-money: our added constraint on the distribution of money, namely that the money consumed together with a particular object is bounded both above and below, and that these bounds may be different for each object. Only Andersson and Svensson (2014) have studied this problem. We shall discuss this paper below, but first let us review what is known from the canonical model. 
In the objects-and-money model, the literature has accepted a weaker notion of Walrasian equilibrium, in which material balance need not be satisfied; it need only be the case that the social endowment is respected. An equilibrium is an allocation, together with a list of prices, one for each object, such that each agent is allocated a favourite bundle from the budget set generated by these prices. When preferences are quasilinear (no wealth effect), Shapley and Shubik (1971) found that the set of prices admitting equilibria form a lattice, and Leonard (1983) found that the equilibria associated with the unique minimal price vector yield the Vickrey rule (also called the pivot mechanism). Demange and Gale (1985) discovered that both the lattice structure and the strategyproofness of the minimal-price rule continue to hold when preferences are not quasilinear. Our rule, $F^{*}$, exploits a similar structure: given a lattice of possible simulated equilibria, it chooses an extremal element. The lemmas used to find the lattice structure in previous models do not apply to ours; we develop new techniques (see section 3.2.1 for a more in-depth discussion of this, and an example of how trying to use the canonical model to solve our problem is sub-optimal).

Some authors have studied the objects-and-money model in the presence of a global budget constraint that must be met with equality: there is a quantity $M \in \mathbb{R}$ of money to be distributed among agents and all of it must be distributed. In this case, efficiency and strategy-proofness together imply extreme inequity. When there are two agents, the only strategy-proof and efficient rules are dictatorial (Schummer (2000)). For more than two agents, Miyagawa (2001), Schummer (2000) and Svensson and Larsson (2002) study the additional axiom of non-bossiness, which requires that no agent can effect a change in the bundle of other agents without also changing his own. Strategyproofness and non-bossiness together almost obviate the role of money; the range of the rule is always finite. The only efficient rules that remain are priority-type mechanisms.

Thus if we want incentive compatibility and any equity we are forced to accept that some resources go unallocated (equivalently, the budget is not balanced), which is why our correspondence $F^{*}$ fails to be fully efficient. For the case when each agent must be allocated an object, Svensson (2009) characterizes the "regular" rules (a range richness condition) that are envy-free and weakly group-strategy-proof. Each such rule associates to each object an upper bound such that the sum of these bounds equals the social endowment of money. The rule is then the most-efficient envy-free allocation that respects these bounds, though it does not generally meet them. Alternatively, we may consider the case when the money is not a social endowment, but rather is supplied by the agents. Moreover, we can assume there is a seller, whose preferences are known, who receives the money collected from agents. The presence of this seller makes efficiency possible. Morimoto 
and Serizawa (2015) then tell us that efficiency, individual rationality, strategy-proofness and no money for losing bidders characterize the min-price Walrasian rule in this environment. This is a generalization of Holmström's (1977) result on quasilinear preferences.

The previous models bound an agent's consumption of money on at most one side. In the usual normalization, agents may consume no more than zero dollars, and must pay money to consume some object. In our model, the consumption of the divisible resource at a given site is bounded on both sides. This may happen in the objects-and-money model if there are price controls; for some exogenous reason, each object has a finite maximum price that may be paid for it. This case is studied by Andersson and Svensson (2014). There, the usual notion of equilibrium often fails to exist: prices do not have sufficient power to influence demand. By not insisting on anonymity or envy-freedom, they can define a type of Walrasian equilibrium that allows for priority based rationing of the objects. Their equilibrium always exists, and for almost all economies there is a unique minimal equilibrium price. On the (generic) sub-domain for which it is defined, the rule that selects the unique minimal price equilibrium is strategy-proof. However, it is important to note that prices do not form a lattice in their model. Thus, our discovery of the lattice structure in our model demonstrates the necessity of developing our own techniques.

Unlike all of the preceding work, our model allows for a single resource pool, which is analogous to an object, to be shared among several agents. One may immediately wonder, then, why not just make "copies" of the pools? We address this further in section 3.2.1, but the short answer is that this will be sub-optimal because we don't know, a priori, how many copies of each object we need. Note that after making copies, we must associate a resource endowment to each copy. Thus, if we make $n$ identical copies, each copied site gets one $n^{\text {th }}$ of the resource. If it turns out that a

particular resource is highly valued by a small number of agents and not valued by others, then we may have done better by making fewer copies of it. It is conceivable that some iterative procedure, where the planner learns the optimal number of copies, reproduces the outcome of our rule. Our approach, however, in addition to finding the best rule and a simple algorithm to compute it, has also uncovered structure that ought prove useful for further analysis.

\section{Model}

There are a finite set $S$ of sites and a finite set $N$ of agents. At each site $s \in S$, there is a social endowment of $e_{s} \in \mathbb{R}_{+}$units of a divisible commodity specific to that site. Each site $s \in S$ has a further constraint: no more than $c_{s} \in \mathbb{N}$ agents may consume there. Assume $\sum_{s \in S} c_{s} \geq|N|$. 
Occasionally it is useful to normalize the endowment so that for each site $s \in S, e_{s}=1$. In this case we write $e \equiv \mathbf{1}$.

Each agent $i \in N$ is assigned to a single site $s \in S$ and given a non-negative quantity $x_{i}$ of that site's commodity. Thus a typical consumption bundle is a pair $\left(x_{i}, s\right) \in \mathbb{R}_{+} \times S$. A typical preference relation is denoted $R_{i}$ with symmetric and asymmetric parts $I_{i}$ and $P_{i}$, respectively. A preference relation $R_{i}$ is increasing if for each pair $x_{i}, y_{i} \in \mathbb{R}$ with $x_{i}>y_{i}$ and for each site $s \in S,\left(x_{i}, s\right) P_{i}$ $\left(y_{i}, s\right)$. The set of continuous, increasing preferences is denoted $\overline{\mathcal{R}}$. If $X$ and $Y$ are subsets of $\mathbb{R}_{+} \times S$, we write $X R_{i} Y$ to mean that for each $\left(x_{i}, s\right) \in X$, and each $\left(y_{i}, t\right) \in Y,\left(x_{i}, s\right) R_{i}\left(y_{i}, t\right)$.

A function $\alpha: N \rightarrow S$ is called a site assignment. ${ }^{2}$ An allocation is a pair $(x, \alpha) \in \mathbb{R}_{+}^{N} \times S^{N}$, $i$ 's bundle being given by $\left(x_{i}, \alpha(i)\right)$. As is standard, $\alpha^{-1}$ denotes the pre-image. Agents are self-centred and so we extend their preferences to the space of allocations in the usual way: $(x, \alpha) R_{i}(y, \beta)$ if and only if $\left(x_{i}, \alpha(i)\right) R_{i}\left(y_{i}, \beta(i)\right)$. An allocation $(x, \alpha)$ is feasible if each site distributes no more commodity than its endowment, and hosts no more agents than its capacity. Formally, for each site $s \in S$,

(Endowment Constraint)

$$
\begin{array}{lc}
\text { (Endowment Constraint) } & \sum_{i \in \alpha^{-1}(s)} x_{i} \leq e_{s}, \\
\text { (Capacity Constraint) } & \left|\alpha^{-1}(s)\right| \leq c_{s} .
\end{array}
$$

Let $\mathcal{R} \subseteq \overline{\mathcal{R}}$ and $\Phi: \mathcal{R}^{N} \rightrightarrows \mathbb{R}^{N} \times S^{N}$. If, for each $R \in \mathcal{R}^{N}$,

- $\Phi(R)$ is a singleton, then it is single-valued,

- $\Phi(R) \neq \emptyset$, then it is non-empty-valued,

- $\Phi(R)$ is a singleton in welfare terms, then it is essentially single valued. ${ }^{3}$

A rule is a non-empty-valued correspondence $\Phi: \mathcal{R}^{N} \rightrightarrows \mathbb{R}^{N} \times S^{N}$ that is essentially single-valued. Given a rule $\Phi$, a selection from $\Phi$ is a function $\varphi: \mathcal{R}^{N} \rightarrow \mathbb{R}^{N} \times S$ such that for each $R \in \mathcal{R}^{N}$, $\varphi(R) \in \Phi(R)$. We write $\varphi \in \Phi$ to indicate that $\varphi$ is a selection from $\Phi$. Since rules are non-emptyvalued, we conflate the function $\varphi: \mathcal{R}^{N} \rightarrow \mathbb{R}^{N} \times S^{N}$ with the single-valued rule $\Phi: \mathcal{R}^{N} \rightrightarrows \mathbb{R}^{N} \times S^{N}$ defined by the equation $\Phi(R)=\{\varphi(R)\}$.

\footnotetext{
$\overline{{ }^{2} \text { For each } i \in N}$, there is $s \in S$ such that $\alpha(i)=s$. Our notation follows Alkan et al. (1991), distinguishing an arbitrary site $s$ from an element of a site assignment, $\alpha(i)$.

${ }^{3}$ For each pair $(x, \alpha)$ and $(y, \beta)$ in $\varphi(R)$, and each agent $i \in N,(x, \alpha) I_{i}(y, \beta)$.
} 
The properties of rules that we study are more easily understood when defined for their selections. Therefore, all properties are defined on single-valued rules and extended as follows: Given a property $\mathbf{P}$ defined for single-valued rules, rule $\Phi$ satisfies $\mathbf{P}$ only if each selection $\varphi \in \Phi$ satisfies $\mathbf{P}$.

\section{Simulated Price Equilibrium.}

We augment the economy with a special divisible commodity that agents may use to purchase their bundles. This commodity is a tool for realizing an allocation and, afterwards, all of it is collected and destroyed. We call this commodity the numeraire.

3.1. Definition. The manager of the commons endows each agent $i \in N$ with $w$ units of the numeraire. We define the demand correspondence $\boldsymbol{D}$ for each preference relation $R_{i} \in \overline{\mathcal{R}}$ and price vector $p \in \overline{\mathbb{R}}^{S}$, by ${ }^{4}$

$$
D\left(R_{i}, p\right):=\left\{\left(x_{i}, s\right): p_{s} x_{i} \leq w \text { and }\left(y_{i}, t\right) P_{i}\left(x_{i}, s\right) \Longrightarrow p_{t} y_{i}>w\right\} .
$$

Denote by $D_{S}$ the projection of the demand correspondence onto the set of sites. The site demand $D_{S}\left(R_{i}, p\right)$ indicates which site assignments are considered best by preference relation $R_{i}$ at prices $p$. Note that it is without loss of generality to assume $w=1$, but we may choose to write $w$ for its semantic value.

The solution concept we study is a form of price equilibrium in which we do not require exact material balance. This is a necessary relaxation of the usual requirements. An implication of Theorem 1 below is that anonymous pricing is incompatible with the allocation of all resources.

Equilibrium: An equilibrium is a list $(p, x, \alpha) \in \mathbb{R}_{++}^{S} \times \mathbb{R}^{N} \times S^{N}$ such that $(x, \alpha)$ is feasible and, for each $i \in N,\left(x_{i}, \alpha(i)\right) \in D\left(R_{i}, p\right)$.

If price vector $p$ admits an equilibrium allocation then we call it an equilibrium price vector. The set of equilibrium price vectors for profile $R$ is $\mathbb{P}(R)$. Equilibrium need not exist, and when it does, it may not be unique. Our main focus is the selection of an equilibrium price vector with beneficial properties, but we take a moment here to introduce two simple properties, each of which, on its own, guarantees that equilibrium exists.

Zero-Commodity Indifference: For each $R_{i} \in \mathcal{R}$, and each pair of sites $s$ and $s^{\prime},(0, s) I_{i}\left(0, s^{\prime}\right)$.

This preference restriction says that if an agent will not consume any resource, regardless of where she goes, then she does not care which site she is assigned. We consider this natural for the

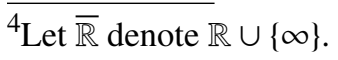


fishing application and many others, like computer time sharing, where there is no value to simply occupying a location. If $\mathcal{R}$ satisfies zero-commodity indifference then the vector of infinite prices $(\infty, \ldots, \infty)$ generates an equilibrium for each profile $R \in \mathcal{R}^{N}$.

It is worthwhile here to introduce a particular class of preferences that satisfy zero-commodity indifference. We say a preference relation $R_{0} \in \overline{\mathcal{R}}$ is linear if there is a vector $v_{0} \in \mathbb{R}^{S}$ such that the function $\left(x_{0}, s\right) \mapsto v_{0 s} x_{0}$, represents $R_{0}$. Let $\mathcal{L} \subset \overline{\mathcal{R}}$ be the set of linear preference relations.

Sufficient Capacity: For each site $s, c_{s} \geq|N|$.

This environment restriction eliminates the capacity constraint. For many fishing problems, where the fishery is large, this is compelling. It may also be compelling in the case of club membership, or computer time sharing, where an agent's presence takes no physical space; only their usage taxes the resource. The sufficient capacity condition guarantees that price vector $p=|N|(w, w, \ldots, w)$ admits an equilibrium with each agent spending his entire endowment $w$ of numeraire to get $1 /|N|$ of some commodity.

When the economy satisfies sufficient capacity, it is easy for us to consider preferences for which there is some value in going to a site, even when no resource is harvested. Consider, for example, a set of mutually exclusive clubs, each of which has money it divides among its members. We may find reason to consider preferences $R_{0} \in \overline{\mathcal{R}}$ for which there is a vector $v \in \mathbb{R}^{S}$ such that the function $(x, s) \mapsto v_{s}+x$ represents $R_{0}$. In this case, $v_{s}$ represents the value of membership in group $s \in S$ and $x$ is the amount of money the agent receives. We call such preferences translation invariant and collect them in set $Q \subseteq \overline{\mathcal{R}}$.

3.2. Minimal Prices. Given an equilibrium price vector $p$, all agents are indifferent between all of the equilibrium allocations it admits. If $p$ and $p^{\prime}$ are equilibrium price vectors and $p<p^{\prime}$, then all agents prefer all of the equilibria at price $p$ to all of the equilibria at price $p^{\prime} .5$ Thus, Paretian social welfare is increasing in the order $<$ on $\mathbb{P}(R)$ and non-decreasing in $\leq$. Given two elements, $p$ and $p^{\prime} \in \mathbb{R}^{S}$, let $p \wedge p^{\prime}$ be the component-wise minimum of $p$ and $p^{\prime}$. That is, for each $s$, $\left(p \wedge p^{\prime}\right)_{s}:=\min \left\{p_{s}, p_{s}^{\prime}\right\}$. If $A \subset \mathbb{R}^{k}$, and if for each pair $\left\{p, p^{\prime}\right\} \subseteq A, p \wedge p^{\prime} \in A$, then we say the pair $(A, \wedge)$ is a lower semi-lattice.

\footnotetext{
${ }^{5}$ The relation $<\subset \mathbb{R}^{S} \times \mathbb{R}^{s}$ is the intersection of the relations $\left\{<\subset \mathbb{R}^{\{s\}} \times \mathbb{R}^{\{s\}}\right\}^{s \in S}$. Thus, $p<p^{\prime}$ if and only if, for each $s \in S, p_{s}<p_{s}^{\prime}$. Likewise for $\leq \subset \mathbb{R}^{S} \times \mathbb{R}^{S}$ and any other binary relation on a product that is derived from marginals.
} 
In the following theorem, we do not assume equilibrium exists. Accordingly, neither zero commodity indifference nor sufficient capacity are assumed. If $\mathbb{P}(R)$ is empty, the theorem holds vacuously.

Theorem 1. For each $R \in \overline{\mathcal{R}}^{N},(\mathbb{P}(R), \wedge)$ is a lower semi-lattice.

Proof. For each $s \in S$, each $\hat{p} \in \mathbb{R}_{+}^{S}$, let $\mathcal{I}_{s}(\hat{p}):=\left\{N^{\prime} \subseteq N: s \in D_{S}\left(R_{i}, \hat{p}\right),|N| w \leq \hat{p}_{s} e_{s},\left|N^{\prime}\right| \leq c_{s}\right\}$. Clearly $\mathcal{M}_{s}(p \wedge q):=\left(N, \mathcal{I}_{s}(p \wedge q)\right)$ is a matroid, whose rank function we denote $\rho_{s}(\cdot){ }^{6}$ Note that $p \wedge q$ admits an equilibrium if and only if the matroids $\left(\mathcal{M}_{s}(p \wedge q)\right)_{s \in S}$ are partitionable.

Let $S_{p}:=\left\{s \in S: p_{s}=(p \wedge q)_{s}\right\}$ and $S_{q}:=\left\{s \in S: q_{s}=(p \wedge q)_{s}\right\}$. Let $N_{p}:=\{i \in N$ : $\left.D\left(R_{i}, p \wedge q\right) \cap S_{q}=\emptyset\right\}$ and $N_{q}:=\left\{i \in N: D\left(R_{i}, p \wedge q\right) \cap S_{p}=\emptyset\right\}$. For each $N^{\prime} \subseteq N$, denote by $\mathcal{M}_{s}(\hat{p}) \backslash N^{\prime}$ the matroid $\mathcal{M}_{s}(\hat{p})$ delete $N^{\prime}$. We now show that if $\left(\mathcal{M}_{s}(p \wedge q) \backslash N_{p}\right)_{s \in S_{q}}$ and $\left(\mathcal{M}_{s}(p \wedge q) \backslash N_{q}\right)_{s \in S_{p}}$ are both partitionable then $\left(\mathcal{M}_{s}(p \wedge q)\right)_{s \in S}$ is partitionable. For each $N^{\prime} \subseteq N$,

$$
\begin{aligned}
\left|N^{\prime} \backslash N_{p}\right|+\left|N^{\prime} \cap N_{p}\right| & \leq \sum_{s \in S_{q}} \rho_{s}\left(N^{\prime} \backslash N_{p}\right)+\sum_{s \in S_{p}} \rho_{s}\left(N^{\prime} \cap N_{p}\right) \\
& =\sum_{s \in S_{q}} \rho_{s}\left(N^{\prime} \backslash N_{p}\right)+\sum_{s \in S \backslash S_{q}} \rho_{s}\left(N^{\prime} \cap N_{p}\right) \leq \sum_{s \in S} \rho_{s}\left(N^{\prime}\right) .
\end{aligned}
$$

The first inequality is by the Edmonds Matroid Partition Theorem. The second equality is because, for each $s \in S_{q}, \rho\left(N_{p}\right)=0$. The third, and final, inequality is by monotonicity of the rank function. The resulting inequality, $\left|N^{\prime}\right| \leq \sum_{s \in S} \rho_{s}\left(N^{\prime}\right)$, implies via Edmond's theorem that $\left(\mathcal{M}_{s}(p \wedge q)\right)_{s \in S}$ is partitionable, proving the claim.

For $i \in N \backslash N_{p}$, there exists $s \in D_{S}\left(R_{i}, p \wedge q\right)$ such that $q_{s}=(p \wedge q)_{s}$. Let $t \in D_{S}\left(R_{i}, q\right)$. Then

$$
\left(\frac{w}{(p \wedge q)_{t}}, t\right) R_{i}\left(\frac{w}{q_{t}}, t\right) R_{i}\left(\frac{w}{q_{s}}, s\right) I_{i}\left(\frac{w}{(p \wedge q)_{s}}, s\right),
$$

and so $t \in D_{S}\left(R_{i}, p \wedge q\right)$. On the other hand, if $r \in S$ satisfies $p_{r}<q_{r}$, then

$$
\left(\frac{w}{q_{s}}, s\right) I_{i}\left(\frac{w}{(p \wedge q)_{s}}, s\right) R_{i}\left(\frac{w}{(p \wedge q)_{r}}, r\right) P_{i}\left(\frac{w}{q_{r}}, r\right)
$$

and therefore $r \notin D_{S}\left(R_{i}, q\right)$. For each $s \in S$, let $\hat{\rho}_{s}$ be the rank function of $\mathcal{M}_{s}(q)$. Let $N^{\prime} \subseteq N \backslash N_{p}$. Line 1 implies, for each $s \in S_{q}$, that $\rho_{s}\left(N^{\prime}\right) \geq \hat{\rho}_{s}\left(N^{\prime}\right)$. Line 2 implies, for each $t \in S \backslash S_{q}$, that $\hat{\rho}_{t}\left(N^{\prime}\right)=0$.

\footnotetext{
${ }^{6}$ See Appendix $\mathrm{C}$ for a brief discussion of matroids.
} 
If $\left(\mathcal{M}_{s}(p \wedge q)\right)_{s \in S}$ is not partitionable, one of the deleted matroid families is not partitionable. Assume without loss of generality that $\left(\mathcal{M}_{s}(p \wedge q) \backslash N_{p}\right)_{s \in S_{q}}$ is not partitionable. Therefore, by Edmonds' theorem, there exists $N^{\prime} \subseteq N \backslash N_{p}$ such that

$$
\left|N^{\prime}\right|>\sum_{s \in S_{q}} \rho_{s}\left(N^{\prime}\right) \geq \sum_{s \in S_{q}} \hat{\rho}_{s}\left(N^{\prime}\right)=\sum_{s \in S_{q}} \hat{\rho}_{s}\left(N^{\prime}\right)+\sum_{s \in S \backslash S_{q}} \hat{\rho}_{s}\left(N^{\prime}\right)=\sum_{s \in S} \hat{\rho}\left(N^{\prime}\right) .
$$

contradicting the assumption that $\left(\mathcal{M}_{s}(q)\right)_{s \in S}$ is partitionable.

Note that by continuity of preferences, $\mathbb{P}(R)$ is closed. Therefore, since it is also bounded below by 0 , Theorem 1 implies $\mathbb{P}(R)$ has a unique smallest element, which we henceforth denote $p^{*}(R)$. Let $\mathcal{A}^{*}(R)$ be the set of site assignments $\alpha$ such that for some $x \in \mathbb{R}^{N},\left(p^{*}(R), x, \alpha\right)$ is an equilibrium.

The Min-Price Rules: Given parameters $c \in \mathbb{Z}_{+}^{S}$ and $e \in \mathbb{R}_{+}^{S}$, let $\mathcal{R} \subseteq \overline{\mathcal{R}}$ satisfy, for each $R \in \mathcal{R}^{N}$, $\mathbb{P}(R) \neq \emptyset$. Let $R \in \mathcal{R}^{N}$ and let $(x, \alpha)$ and $(y, \beta)$ be two equilibria supported by price $p^{*}(R)$. Then $(x, \alpha)$ and $(y, \beta)$ are Pareto indifferent for profile $R$. It follows that the mapping $F^{*}: \mathcal{R}^{N} \rightrightarrows \mathbb{R}_{+}^{S} \times S^{N}$ given for each profile $R \in \mathcal{R}^{N}$ by

$$
F^{*}(R)=\left\{(x, \alpha): \alpha \in \mathcal{A}^{*}(R), x_{i}=\frac{w}{p_{\alpha(i)}^{*}(R)}\right\}
$$

is a rule. We call $F^{*}$ the min-price rule on $\mathcal{R}^{N}$.

3.2.1. Discussion: Why lattice results of previous work do not apply here. Lattice structures have been observed in many discrete assignment models, with and without the presence of divisible resources. For objects-and-money, Demange and Gale's (1985) model of one-to-one matching is readily adapted to the case where one side is not agents but objects. We show here that previous techniques cannot successfully be applied this model.

When confronted with our problem, one might first attempt to solve it by, ex ante, making "copies" of the sites. The following example shows this approach fails.

Example 1. Let $S:=\{a, b\}, N:=\{1,2,3\}, e:=(1,1)$, and $c:=(3,2)$. Let $R_{0} \in \overline{\mathcal{R}}^{N}$ satisfy $(1 / 3, a) P_{0}$ $(1, b)$. Clearly, the min-price equilibrium of the profile $\left(R_{0}, R_{0}, R_{0}\right)$ has all agents consuming at site $a$. Moreover, it must have all agents consuming the same quantity. Thus, to find this equilibrium via the "making copies" method, we must make three copies of site $a,\left\{a^{1}, a^{2}, a^{3}\right\}$, and give each $a^{k}$ endowment $e_{a} / 3$. 
Consider a preference relation $R_{0}^{\prime}$ that is indifferent between consumption at sites $a$ and $b$ : for each $x \in \mathbb{R},(x, a) I_{0}^{\prime}(x, b)$. It is easy to verify that the minimal equilibrium price for profile $\left(R_{0}^{\prime}, R_{0}^{\prime}, R_{0}^{\prime}\right)$ is $(2,2)$. With this price, at equilibrium, an agent goes to site $a$ and consumes $1 / 2$. However, we deduced above that, by making ex ante copies, the most he could consume is 1/3.

Thus, making ex-ante copies sometimes prevents us from finding the truly minimal price, and so a new approach is necessary. However, we could perhaps have a simpler proof by the proper use of "copies" to show the lattice property. We would begin with two equilibrium prices $p$ and $q$ and attempt to show that $p \wedge q$ is an equilibrium price. The Demange and Gale proof begins with the following Decomposition Lemma. ${ }^{7}$

Lemma (Demange and Gale). Let $(p, x, \alpha)$ and $(q, y, \beta)$ be equilibria for economy $R \in \mathcal{R}^{N}$. Define the following sets:

$$
\begin{array}{ll}
N^{p}:=\left\{i \in N:(x, \alpha) P_{i}(y, \beta)\right\} & S^{q}:=\left\{s \in S: p_{s}>q_{s}\right\} \\
N^{q}:=\left\{i \in N:(y, \beta) P_{i}(x, \alpha)\right\} & S^{p}:=\left\{s \in S: q_{s}>p_{s}\right\} \\
N^{=}:=\left\{i \in N:(x, \alpha) I_{i}(y, \beta)\right\} & S^{=}:=\left\{s \in S: p_{s}=q_{s}\right\} .
\end{array}
$$

Then $\alpha\left(N^{p}\right) \subseteq S^{q}, \beta\left(N^{p}\right) \subseteq S^{q}, \alpha\left(N^{q}\right) \subseteq S^{p}$ and $\beta\left(N^{q}\right) \subseteq S^{p}$.

In fact, this lemma fails in our class of models, as we show with the following example.

Example 2. The environment is the same as in Example 1, except we set $e=(\lambda, 1)$, with $\lambda>1$. Assuming $w=1$ (otherwise, renormalize), we may be sure that, for each site $s \in S$, we need $\left\lfloor(p \wedge q)_{s}\right\rfloor$ copies to find an equilibrium supported by $p \wedge q$.

Let $R \in \mathcal{L}^{N}$ be a profile of linear preferences with preference coefficients $v_{1}=(1,1 / 2), v_{2}=$ $(1 / 2, \lambda)$, and $v_{3}=(1,1)$. Let $p=(4,2)$ and $q=\left(2 \lambda^{-1}, 4\right)$. Clearly, $D_{S}\left(R_{1}, q\right)=\{a\}$, and since

$$
v_{1, a} \cdot \frac{w}{p_{a}}=1 \cdot \frac{1}{4}=\frac{1}{2} \cdot \frac{1}{2}=v_{1, b} \cdot \frac{w}{p_{b}},
$$

$D_{S}\left(R_{1}, p\right)=\{a, b\}$. Symmetrically, $D_{S}\left(R_{2}, p\right)=\{b\}$ and, since

$$
v_{2, b} \cdot \frac{w}{q_{b}}=\lambda \cdot \frac{1}{4}=\frac{1}{2} \cdot \frac{\lambda}{2}=v_{2, a} \cdot \frac{w}{q_{a}},
$$

$D_{S}\left(R_{2}, p\right)=\{a, b\}$. Two site assignments $\alpha$ and $\beta$ are given in the following table:

\footnotetext{
${ }^{7}$ The Lemma as stated is the combination of Lemma 1 and Corollary 1 in Demange and Gale (1985).
} 


\begin{tabular}{c|c|c|c|} 
& 1 & 2 & 3 \\
\hline$\alpha$ & $a$ & $b$ & $b$ \\
\hline$\beta$ & $a$ & $b$ & $a$ \\
\hline
\end{tabular}

It is clear that $\alpha$ is an equilibrium assignment for price $p$ and $\beta$ is an equilibrium assignment for price $q$. The Decomposition Lemma is violated as $3 \in N^{q}$, but $\alpha(3)=b \in S^{p}$ and $\beta(3)=a \in S^{q}$.

\subsection{Properties of Min-price Rules.}

3.3.1. Seeking Min-price Equilibria: Necessary and Sufficient Conditions. If the resource of a site is completely distributed, we say the site is exhausted. Theorem 2 below implies that, at any min-price equilibrium, at least one site is exhausted. What prevents all sites from being exhausted are the preference relations of those consuming at exhausted sites. To see why, consider a simple example.

Example 3. Let $S:=\{a, b\}, N:=\{1,2,3\}, e:=(1,1)$, and $c:=(3,2)$. For the simulated market, give each agent $w=1$ unit of numeraire. Let $R \in \mathcal{L}^{N}$ be a profile of linear preferences with preference coefficients $v_{1}=(3,1), v_{2}=(1,1)$, and $v_{3}=(1,3)$. The minimal equilibrium price vector for this profile is $(2,2)$ and note that $D_{S}\left(R_{2},(2,2)\right)=\{a, b\}$. Consider site assignment $\alpha \in S^{N}$ given by $\alpha(1)=\alpha(2)=a$ and $\alpha(3)=b$. Site $a$ is exhausted, while agent 3 consumes only half of site $b$ 's endowment. We would therefore like to lower the price of site $b$. For $\varepsilon>0$, consider price vector $(2,2-\varepsilon)$. At this price, agents 1 and 3 still choose to consume at sites $a$ and $b$ respectively. However, agent 2 is no longer indifferent; $D_{S}\left(R_{2},(2,2-\varepsilon)\right)=\{a\}$. Then the consumption of site $a$ 's resource is $2\left(\frac{w}{2-\varepsilon}\right)=\frac{2}{2-\varepsilon}>1=e_{a}$, violating feasibility.

The tension in the previous example can be transmitted via chains of indifference to economies of arbitrary size. These chains decide the equilibrium price list. Formally, let $(x, \alpha) \in F^{*}(R)$. If there is a statement of the form

$$
\left(x_{i^{1}}, \alpha\left(i^{1}\right)\right) I_{i^{1}}\left(x_{i^{2}}, \alpha\left(i^{2}\right)\right) I_{i^{2}} \cdots I_{i^{n}}\left(x_{i}, \alpha(i)\right),
$$

then site $\alpha(i)$ is blocked via indifference by site $\alpha\left(i^{1}\right)$. Theorem 2 below shows that each site is blocked via indifference by an exhausted site, where we include in this terminology the case that the site itself is exhausted.

Given prices $p$, let $\tilde{c}_{s}(p) \in \mathbb{Z}$ denote the integer satisfying

$$
w \tilde{c}_{s}(p) \leq p_{s} e_{s}<w\left(\tilde{c}_{s}(p)+1\right) .
$$


The implied capacity of site $s$ given prices $p$, defined as $c_{s}(p):=\min \left\{c_{s}, \tilde{c}_{s}(p)\right\}$, is the largest number of agents that can be assigned to $s$ under price $p_{s}$. Note that if $p_{s} e_{s}<w$ then $c_{s}(p)=0$.

Only if $w c_{s}(p)=p_{s} e_{s}$ is it possible for $s$ to be exhausted at $p$. We will refer to such sites as having endowment-divisible value. Given $p \in \mathbb{P}(R)$, say that $\alpha \in N^{S}$ is balanced if, for each site $s$ having endowment-divisible value, $\left|\alpha^{-1}(s)\right| \geq c_{s}(p)-\mathbb{1}\left[c_{s} \geq \tilde{c}_{s}(p)\right]$, and for every other site $t$, $\left|\alpha^{-1}(t)\right|=c_{t}(p)$. An equilibrium is balanced if its associated site-assignment is balanced.

Theorem 2. If $F^{*}(R)$ is non-empty, then it contains a balanced equilibrium. At each $(x, \alpha) \in F^{*}(R)$, whether balanced or not, each site is blocked via indifference by an exhausted site. Finally, if $(p, x, \alpha)$ is a balanced equilibrium for economy $R \in \overline{\mathcal{R}}^{N}$ such that each site is blocked via indifference by an exhausted site, then $(x, \alpha) \in F^{*}(R)$.

3.3.2. Welfare and comparative statics. As is typical of incentive compatible rules, the outcome of a min-price rule is not always Pareto efficient. It is efficient in a limited sense: there is no benefit to ex post re-trading among the agents. Unfortunately, for many preference profiles, there will be no allocations in $F^{*}$ that distribute all of the social endowment. When the economy satisfies sufficient capacity, we can calculate the quantity of undistributed resources and find that it is small, asymptotically. Moreover, this lack of efficiency is not novel among social choice rules. Recall that Vickrey-Clarke-Groves mechanisms are typically not budget balanced.

We use balanced assignments to calculate the undistributed resources for economies that satisfy sufficient capacity. At a balanced, min-price allocation, the amount discarded of each resource is bounded above by $e_{s} / k+1$ if $k$ people consume the resource.

Theorem 3. Assume sufficient capacity. For each profile $R \in \overline{\mathcal{R}}^{N}$, there is an allocation $(x, \alpha) \in$ $F^{*}(R)$ such that for each site $s$,

$$
\sum_{i \in \alpha^{-1}(s)} x_{i} \geq\left(\frac{\left|\alpha^{-1}(s)\right|}{\left|\alpha^{-1}(s)\right|+1}\right) e_{s} .
$$

Proof. Since we assume sufficient capacity, $F^{*}$ is defined on $\overline{\mathcal{R}}^{N}$. Let $(x, \alpha) \in F^{*}(R)$ be such that $\alpha$ is balanced. Let $p=p^{*}(R)$. If site $s$ has endowment-divisible value at $p$, then since $\alpha$ is balanced, $\left|\alpha^{-1}(s)\right|+1 \geq c_{s}(p)$, and endowment-divisibility then yields $w c_{s}(p)=p_{s} e_{s}$. Thus, $\left|\alpha^{-1}(s)\right|+1 \geq p_{s} e_{s} / w$. Since $\left(\left|\alpha^{-1}(s)\right| w\right) p_{s}^{-1}=\sum_{i \in \alpha^{-1}(s)} x_{i}$, dividing both sides by $\left|\alpha^{-1}(s)\right|$ gives

$$
\frac{\left|\alpha^{-1}(s)\right|+1}{\left|\alpha^{-1}(s)\right|} \geq \frac{e_{s}}{\sum_{i \in \alpha^{-1}(s)} x_{i}} .
$$


If site $s$ is not endowment-divisible, $\left|\alpha^{-1}(s)\right|=c_{s}(p)$ and so

$$
\left|\alpha^{-1}(s)\right|+1=c_{s}(p)+1>\frac{p_{s} e_{s}}{w} .
$$

Again dividing through by $\left|\alpha^{-1}(s)\right|$ gives the result.

We now analyse the response of prices to changes in preferences, for which we no longer need to assume sufficient capacity. The result is a theorem that provides useful tools for comparative statics. We later use these tools to demonstrate the incentive properties of min-price rules.

For analytical precision, we confine ourselves to a class of preference transformations that represent an unambiguous strengthening or weakening of preference for a given site or set of sites. Let $R_{i} \in \overline{\mathcal{R}}$ and $\hat{S} \subseteq S$. For each $d \in \mathbb{R}$, define $\boldsymbol{R}_{i}^{\hat{S}, d}$ so that $\left.R_{i}^{\hat{S}, d}\right|_{\mathbb{R} \times S \backslash \hat{S}}=\left.R_{i}\right|_{\mathbb{R} \times S \backslash \hat{S}}$ and for each $\hat{s} \in \hat{S}$ and each $t \in S \backslash \hat{S}$,

$$
(x, \hat{s}) R_{i}(y, t) \Longrightarrow(x-d, \hat{s}) R_{i}^{\hat{S}, d}(y, t) .
$$

We say that $R_{i}^{\hat{S}, d}$ is a site-translation through set $\hat{S}$, or an $\hat{S}$-translation, of $R_{i}$. If $\hat{S}=\{s\}$, we simply write $s$-translation. If $d>0$ we call the translation positive. We consider this the positive direction because it represents an increased preference for $s$ relative to other sites. In fact, for any $x_{i} \in \mathbb{R}$ and any $s \in S$, the lower contour set of $R_{i}^{s, d}$ at $\left(x_{i}, s\right)$ contains the lower contour set of $R_{i}$ at $\left(x_{i}, s\right)$.

Note that, generally, $R_{i} \in \mathcal{R}$ does not guarantee $R_{i}^{s, d} \in \mathcal{R}$. In particular, when $\mathcal{R}$ satisfies zero commodity indifference, the only translations that remain in the domain are the identity translations. However, it is often the case that, if $F^{*}$ is defined on domain $\mathcal{R}^{N}$, then it is also defined on $\left(\mathcal{R} \cup\left\{R_{i}^{s, d}\right\}\right)^{N}$. As this subsection and the next study the properties of $F^{*}$, we welcome any expansion of the domain, as it is better to show more general properties. In the next section, when we characterize $F^{*}$, the opposite is true: the smaller the domain on which $F^{*}$ is the unique rule of interest, the more powerful the result.

We collect in a lemma some important properties of $p^{*}$.

Lemma 1. Fix $i \in N$ and $s \in S$. Assume $\mathbb{P}(R)$ is non-empty. Define the function $\pi$ for each $a \in \mathbb{R}$ by $\pi(a):=p^{*}\left(R_{i}^{s, a}, R_{-i}\right)$.

Property 1: $\pi$ is non-decreasing where defined

Property 2: If $s \notin D_{S}\left(R_{i}, p^{*}(R)\right)$, then there exists $\bar{d}>0$ such that for each $d<\bar{d}, \pi(d)=\pi(0)$. 
The following lemma, proved in the appendix, is important in showing that groups cannot manipulate min-price rules. In particular, no group of agents can lower the price of all the sites at which the consume, though they may be able to lower the prices of some.

Lemma 2. Let $N^{\prime} \subseteq N, R \in \overline{\mathcal{R}}^{N}$, and $R_{N^{\prime}}^{\prime} \in \mathcal{R}^{N^{\prime}}$. Assume $\mathbb{P}(R)$ and $\mathbb{P}\left(R_{N^{\prime}}^{\prime}, R_{N \backslash N^{\prime}}\right)$ are non-empty. Let $\alpha \in \mathcal{A}^{*}\left(R_{N^{\prime}}^{\prime}, R_{N \backslash N^{\prime}}\right)$ and $d:=2 \max _{s \in S}\left\{e_{s}\right\}$. Then $p^{*}\left(\left(R_{j}^{\alpha(j), d}\right)_{j \in N^{\prime}}, R_{N \backslash N^{\prime}}\right)$ is defined and there is a site $t \in \alpha\left(N^{\prime}\right)$ such that

$$
p_{t}^{*}\left(\left(R_{j}^{\alpha(j), d}\right)_{j \in N^{\prime}}, R_{N \backslash N^{\prime}}\right) \geq p_{t}^{*}(R)
$$

3.3.3. Incentive Compatibility. Let $\mathcal{R}^{N}$ be a domain on which $F^{*}$ is defined. The following incentive compatibility property is standard: no group of agents should strictly benefit by reporting false preferences.

Weak Group-strategy-proofness (w-GStP): For each $R \in \mathcal{R}^{N}$, each group $N^{\prime} \subseteq N$, and each partial profile of preferences $\hat{R}_{N^{\prime}}:=\left(\hat{R}_{i}\right)_{i \in N^{\prime}} \in \mathcal{R}^{N^{\prime}}$, there is an agent $k \in N^{\prime}$ such that

$$
\varphi_{k}(R) R_{k} \varphi_{k}\left(\hat{R}_{N^{\prime}}, R_{N \backslash N^{\prime}}\right) \text {. }
$$

The tools of Lemmas 1 and 2 make intuitive why min-price rules should have such nice incentive properties. Agents have limited influence over the prices of each site, and what influence they do have is the "appropriate" kind. Most crucially, we show that if an agent causes the price of a site to decrease, then the agent must abandon consumption at this site. This is due to the indivisibilities in the problem and is a major difference between equilibrium here and equilibrium in classical exchange economies.

Theorem 4. $F^{*}$ is weakly group-strategy-proof.

Proof. In what follows, the preferences of agents $N \backslash N^{\prime}$ are held constant and therefore we suppress their notation.

Let $f \in F^{*}$, and $R \in \mathcal{R}^{N}$. To arrive at a contradiction, assume there is a set $N^{\prime} \subseteq N$ and a partial profile $\hat{R}:=\left(\hat{R}_{j}\right)_{j \in N^{\prime}}$ such that for each $k \in N^{\prime}$,

$$
\left(x_{k}, \alpha(k)\right):=f_{k}(\hat{R}) \quad P_{k} \quad f_{k}(R) .
$$

Note that $f(\hat{R})$ is an equilibrium for $R^{d}:=\left(R_{j}^{\alpha(j), d}\right)_{j \in N^{\prime}}$. Therefore $p^{*}\left(R^{d}\right) \leq p^{*}(\hat{R})$. Since $d$ is large, each $k \in N^{\prime}$, with preferences $R_{k}^{d}$, will feasibly choose only $\alpha(k)$ at prices $p^{*}\left(R^{d}\right)$. Thus, for 
each $k \in N^{\prime}$, there is $\bar{x}_{k}$ satisfying $\bar{x}_{k} \geq x_{k}$ such that

$$
f_{k}\left(R^{d}\right)=\left(\bar{x}_{k}, \alpha(k)\right) .
$$

Therefore, $R^{d}$ is also a joint manipulation for group $N^{\prime}$. Now we apply Lemma 2: there is a site $t \in(\alpha(j))_{j \in N^{\prime}}$ such that

$$
p_{t}^{*}\left(R^{d}\right) \geq p_{t}^{*}(R)
$$

a contradiction.

\section{Characterization}

In this section we provide a characterization of min-price rules in terms of appealing properties. Our primary focus is on preference elicitation in the presence of a weakened form of anonymity. We show that, if $\mathcal{R}$ is sufficiently rich, then $F^{*}$ is a supercorrespondence of any rule satisfying the following properties. Recall here that we define properties on single-valued rules and say that a correspondence satisfies a property if all its selections do.

The first property is implied by, and is much weaker than, weak group-strategy-proofness.

Strategy-proofness (StP): For each $R \in \mathcal{R}^{N}$, each $i \in N$, and each preference relation $\hat{R}_{i} \in \mathcal{R}$,

$$
\varphi_{i}(R) R_{i} \varphi_{i}\left(\hat{R}_{i}, R_{-i}\right) .
$$

In this model, as in many others, we may adapt the usual sequential priority procedure to both elicit preferences truthfully and achieve Pareto efficiency. The inequity of such rules is extreme and therefore they are inappropriate for the applications we have envisioned. We insist upon a form of anonymity that requires an agent's welfare depend only on his preferences and the unordered list of preference relations present in the economy. Note that this is weaker than the usual form, which requires an agent's bundle depend only on his preferences and the unordered list of preference relations present in the economy.

Welfare Anonymity (W-Anon): Let $R \in \mathcal{R}^{N}$ and let $\sigma: N \rightarrow N$ be a bijection. Let $i \in N$ and $\sigma(j)=i$. Then

$$
\varphi_{i}(R) I_{i} \varphi_{j}\left(\left(R_{\sigma(k)}\right)_{k \in N}\right) .
$$

Since the consumption space is compact and preferences continuous, the Hausdorff distance $H^{\Delta}$ between the graphs of any pair $R$ and $R^{\prime}$ of preference relations generates a metric topology on the space of continuous preference relations. The following property is very mild, as it requires 
continuity in welfare space only in special cases. Consider a convergent sequence of profiles. Suppose the rule chooses the same allocation for all of the profiles on the sequence. Then in the limit profile, the agents are indifferent between what the rule chooses at the limit and what it has chosen all along the sequence.

Constant Sequence Welfare Continuity (w-Cont): Let $\left(R^{n}\right)^{n \in \mathbb{N}} \subset \mathcal{R}^{N}$ be a sequence converging to $R$. Assume there is an allocation $(x, \alpha)$ such that for each $n \in \mathbb{N}, \varphi\left(R^{n}\right)=(x, \alpha)$. Then for each agent $i \in N,\left(x_{i}, \alpha(i)\right) I_{i} \varphi_{i}(R)$.

In a model as rich as this, we should not expect $S t P, W$-Cont, and $W$-Anon to identify a single rule. Rather than introduce further properties, however, we study the consequences of a rule being the most efficient, in a point-wise sense, in a class of rules. In general, we may define

Strong Undomination in $\mathfrak{C}$ : Fix a class of rules $\mathfrak{C}$. Say that $\varphi$ is strongly undominated in $\mathfrak{C}$ if for each $\psi \in \mathbb{C}$ and each $R \in \mathcal{R}^{N}$

$$
\psi_{i}(R) P_{i} \varphi_{i}(R) \Longrightarrow \exists j, \varphi_{j}(R) P_{j} \psi_{j}(R)
$$

We require that $\varphi$ be strongly undominated in the class of rules satisfying our previous properties.

Strong Undomination in W-Anon, StP, w-Cont: Rule $\varphi$ is strongly undominated in the class of rules defined on $\mathcal{R}^{N}$ satisfying welfare anonymity, strategy-proofness, and constant sequence continuity. Henceforth we refer to this property simply as strong undomination.

The smaller the set $\mathcal{R}$, the less bite our first three conditions have. If $\mathcal{R}$ is a singleton, then in fact strategy-proofness and constant sequence continuity are vacuous. Such is clearly not an interesting case. Instead, make the following assumptions about $\mathcal{R}$ :

(1) For each $R \in \mathcal{R}^{N}, \mathbb{P}(R) \neq \emptyset$.

(2) For each $R \in \mathcal{R}^{N}$, there is $R_{0}^{p^{*}(R)} \in \mathcal{R}$ such that $D_{S}\left(R_{0}^{p^{*}(R)}, p^{*}(R)\right)=S$. In general, we use $R_{0}^{p}$ when discussing a preference relation with the property that $D_{S}\left(R_{0}^{p}, p\right)=S$.

(3) Let $R \in \mathcal{R}^{N}, i \in N$, and $A \subseteq D\left(R_{i} ; p^{*}(R)\right)$. Denote by $R_{i}^{-1}[(x, s)]$ and $R_{i}[(x, s)]$ the upper and lower contour sets, respectively, of $R_{i}$ at $(x, s)$. Let $\mathcal{T}\left(R_{i}, A\right)$ be the set of preference relations $\hat{R}_{i} \in \overline{\mathcal{R}}$ such that, for each $(x, s) \in A, \hat{R}^{-1}[(x, s)] \cap R[(x, s)]=A$. For each $\varepsilon>0$, there is $R_{i}^{\varepsilon} \in \mathcal{T}\left(R_{i}, A\right)$ such that $H^{\Delta}\left(R_{i}, \hat{R}_{i}\right)<\varepsilon$ and $\hat{R}_{i} \in \mathcal{R}$.

Since $\mathcal{R}$ satisfies 1,2 , and 3 , we say it is sufficiently rich. Note that the third assumption amounts to ensuring that the domain has sufficiently many Maskin monotonic transformations. The linear 
domain $(\mathcal{L})$ is sufficiently rich. The translation invariant domain $(Q)$ satisfies all the requirements except 1 . Thus if the environment has sufficient capacity, $Q$ is sufficiently rich. However, this is not necessary; subsets of $Q$ for which the preference coefficients are bounded can also be sufficiently rich.

We may now state the characterization, the proof of which is in the appendix.

Theorem 5. A rule $\Phi$ on $\mathcal{R}^{N}$ is strategy-proof, welfare anonymous, constant sequence continuous, and strongly undominated in these properties if and only if it is a subcorrespondence of $F^{*}$.

This characterization is tight. To show the independence of constant sequence continuity, we may adapt the rule used to similar effect in Tierney (2016). ${ }^{8}$

\section{Simulated Auction}

In this section we verify that our rule can be calculated easily in practice via an extensive form bidding process. An auction is a type of game form used to implement allocation rules that are based on prices. The messages of an auction are demand schedules. In this model, a demand schedule for agent $i$ is a function $D_{i}: \mathbb{R}_{+}^{S} \rightarrow 2^{S}$ such that there exists $R_{i} \in \mathcal{R}$ satisfying $D_{i}(\cdot)=D_{S}\left(R_{i}, \cdot\right)$. The set of demand schedules is $\mathcal{D}$. It is desirable that, rather than reporting their entire demand schedule, agents instead report their demands in response to a smaller list of prices. Typically, a price $q \in \mathbb{R}^{S}$ is announced and the reported demands, $\left(D_{i}(q)\right)_{i \in N}$, then determine the next price asked, thus making the auction game an extensive form. We shall assume that the auction proceeds in continuous time and that the price dynamic is described by a differential equation. Let $\delta>0$. We propose a price dynamic whose time derivative satisfies, for each point in time $\tau \in \mathbb{R}$, each demand profile $D \in \mathcal{D}^{N}$, and each site $s \in S, \dot{p}_{s}(D, \tau) \in\{0, \delta\}$. Therefore, to define $\dot{p}$ it remains to determine the rule for choosing which set of sites will have their prices rising.

Fix $D \in \mathcal{D}^{N}$. For each site $s \in S$, each $N^{\prime} \subseteq N$, and each $q \in \mathbb{R}^{S}$, let

$$
\rho_{s}\left(N^{\prime}, q\right):=\min \left\{\left|\left\{i \in N^{\prime}: s \in D_{i}(q)\right\}\right|, c_{s}(q)\right\} .
$$

A set of sites $\hat{S} \subseteq S$ is overdemanded at $q$ if the set $\hat{N}:=\left\{i \in N: D_{i}(q) \subseteq \hat{S}\right\}$ satisfies $|\hat{N}|>$ $\sum_{s \in \hat{S}} \rho_{s}(\hat{N}, q)$. Let $N^{\prime} \subseteq N$ be arbitrary. Let $S^{\prime}:=\cup_{i \in N^{\prime}} D_{i}(q)$. If there are no overdemanded sites

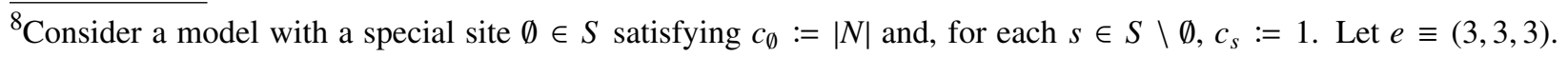
Consider a restricted preference domain $\mathcal{V} \subseteq Q$ such that for each $v_{0} \in \mathcal{V}, v_{0 \emptyset}=0$, and for each $s \in S \backslash \emptyset, v_{0 s} \in(0,3)$. 
at $q$,

$$
\left|N^{\prime}\right| \leq \sum_{s \in S^{\prime}} \rho_{s}\left(N^{\prime}, q\right)=\sum_{s \in S} \rho_{s}\left(N^{\prime}, q\right),
$$

where the equality follows from the fact that, for each $r \in S \backslash S^{\prime}, \rho_{r}\left(N^{\prime}, q\right)=0$. Thus, Edmond's Matroid Partition Theorem implies that $q$ is an equilibrium price satisfying demands $\left(D_{i}(q)\right)_{i \in N}$.

The auction will proceed by raising the price of some, but not all overdemanded sets. In particular, a set $\hat{S} \subseteq S$ is minimally overdemanded if it is overdemanded and if, for each $\tilde{S} \subsetneq \hat{S}, \tilde{S}$ is not overdemanded. A site $s \in S$ is minimally overdemanded if it is a member of a minimally overdemanded set. Define

$$
\Delta_{S}(D, q):= \begin{cases}\delta & s \text { is minimally overdemanded } \\ 0 & \text { otherwise. }\end{cases}
$$

Let $R \in \mathcal{R}^{N}$ and, for each $i \in N$, let $D_{i}(\cdot):=D_{S}\left(R_{i}, \cdot\right)$. Let $p: \mathfrak{D}^{N} \times \mathbb{R} \rightarrow \mathbb{R}^{S}$ be given by the differential equation $\dot{p}(D, \tau)=\Delta(D, p(\tau))$ with initial condition given for each $s \in S$ by $p_{s}(0)=w / e_{s}$. . We now show that the price dynamic thus defined converges in finite time to the minimal Walrasian price.

Proposition 1. For each $D \in \mathfrak{D}$, there exists $T \in \mathbb{R}_{+}$such that $p(D, T)=p^{*}(R)$.

Proof. We first prove the following claim:

Claim 1. For each $\tau \in \mathbb{R}, p(\tau) \leq p^{*}(R)$.

Proof. Let $p \leq p^{*}(R)$ and let $S^{e q}:=\left\{s \in S: p_{s}=p_{s}^{*}(R)\right\}$. Let $N^{e q}:=\left\{i \in N: D_{i}(p) \cap S^{e q} \neq \emptyset\right\}$. If $s \in S^{e q}$ and $s \in D_{i}(p)$ then $s \in D_{i}\left(p^{*}(R)\right)$. Therefore, for each $N^{\prime} \subseteq N^{e q}$ and each $s \in S^{e q}$, $\rho_{s}\left(N^{\prime}, p\right)=\rho_{s}\left(N^{\prime}, p^{*}(R)\right)$. For each $r \notin S^{e q}, \rho_{r}\left(N^{\prime}, p^{*}(R)\right)=0$. Let $S^{\prime} \subseteq S^{e q}$ and $N^{\prime}:=\{i \in N$ : $\left.D_{i}(p) \subseteq S^{\prime}\right\}$. Since $N^{\prime} \subseteq N^{e q}$, if it were the case that $S^{\prime}$ is overdemanded at $p$, then

$$
\left|N^{\prime}\right|>\sum_{s \in S^{\prime}} \rho_{s}\left(N^{\prime}, p\right)=\sum_{s \in S} \rho\left(N^{\prime}, p^{*}(R)\right)
$$

contradicting, via the Matroid Partition Theorem, the fact that $p^{*}(R)$ is an equilibrium price. Thus, neither $S^{e q}$ nor any of its subsets are overdemanded at $p$.

\footnotetext{
${ }^{9}$ In fact, the proof below makes plain that any initial condition $p(0) \leq p^{*}(R)$ will work, so if the market manager is certain of a lower bound on $p^{*}(R)$, she may start the auction there.
} 
We must also show that $S^{e q}$ does not belong to any minimally overdemanded set. Let $N^{\prime} \subseteq N^{e q}$. Since $p^{*}(R)$ is an equilibrium price we deduce

$$
\left|N^{\prime}\right| \leq \sum_{s \in S} \rho_{s}\left(N^{\prime}, p^{*}(R)\right)=\sum_{s \in S^{e q}} \rho_{s}\left(N^{\prime}, p^{*}(R)\right)=\sum_{s \in S^{e q}} \rho_{s}\left(N^{\prime}, p\right),
$$

where the initial inequality is from the Matroid Partition Theorem. Now let $\hat{S} \supseteq S^{e q}$ and $\hat{N}:=$ $\left\{i \in N: D_{i}(p) \subseteq \hat{S}\right\}$. If $s \in S^{e q}$, then $\rho_{s}(\hat{N}, p)=\rho_{s}\left(\hat{N} \cap N^{e q}, p\right)$. Therefore, if $\hat{S}$ is overdemanded, line 3 and the Matroid Partition Theorem yield

$$
\begin{aligned}
|\hat{N}| & >\sum_{s \in S \backslash S^{e q}} \rho_{s}(\hat{N}, p)+\sum_{s \in S^{e q}} \rho_{s}(\hat{N}, p) \\
& =\sum_{s \in S \backslash S^{e q}} \rho_{s}(\hat{N}, p)+\sum_{s \in S^{e q}} \rho_{s}\left(\hat{N} \cap N^{e q}, p\right) \\
& \geq \sum_{s \in S \backslash S^{e q}} \rho_{s}(\hat{N}, p)+\left|\hat{N} \cap N^{e q}\right| .
\end{aligned}
$$

Therefore, $\left|\hat{N} \backslash N^{e q}\right|>\sum_{s \in S \backslash S^{e q}} \rho_{s}(\hat{N}, p) \geq \sum_{s \in S \backslash S^{e q}} \rho_{s}\left(\hat{N} \backslash N^{e q}, p\right)$, where the last inequality is by monotonicity of $\rho(\cdot, p)$. We conclude that $S \backslash S^{e q}$ is overdemanded and, furthermore, that for each $s \in S^{e q}, s$ is not minimally overdemanded.

Since the price path $p(\cdot)$ is continuous and since $p(0)=w^{-1} e$, if there exist $\tau \in \mathbb{R}$ and $s \in S$ such that $p_{s}(\tau)>p_{s}^{*}(R)$, then there are $\tau^{\prime}<\tau$ and $s^{\prime} \in S$ such that $p\left(\tau^{\prime}\right) \leq p^{*}(R)$ and $p_{s^{\prime}}\left(\tau^{\prime}\right)=p_{s^{\prime}}^{*}(R)$. What we deduced in the preceding paragraphs then implies that for $\tau^{\prime \prime} \geq \tau^{\prime}, \dot{p}_{s^{\prime}}\left(D, p\left(\tau^{\prime \prime}\right)\right)=0$, a contradiction.

Note that if $\tau^{\prime}>\tau$ and $p\left(\tau^{\prime}\right)=p(\tau)$, then $p(t) \in \mathbb{P}(R)$. Contrapositively, if $p(\tau) \notin \mathbb{P}(R)$, then for each $\tau^{\prime}>\tau, p\left(\tau^{\prime}\right) \gtrless p(\tau)$, and the rate of increase is bounded away from zero. Therefore, since $p(\cdot)$ is bounded above by $p^{*}(R)$, there is some finite $T \in \mathbb{R}$ such that for each $\tau^{\prime}>T, p\left(\tau^{\prime}\right)=p(T)$. It follows that $p(T) \in \mathbb{P}(R)$, so $p(T) \geq p^{*}(R) \geq p(T)$ and the result follows.

The following theorem is now an immediate consequence of the Revelation Principle:

Theorem 6. The auction using price dynamic $p(\cdot)$ implements $F^{*}$ in dominant strategies.

\section{Extension: Personal Endowment of Money}

We now allow for agents to purchase the resource with their own money. Thus, each agent $i \in N$ has an endowment $w_{i}>0$ of money. A typical consumption bundle is a triple $\left(m_{i}, x_{i}, s\right) \in \mathbb{R}^{2} \times S$, 
where $m_{i}$ is his consumption of money. Notation for a typical preference relation is unchanged. Assume that preferences are increasing: if $\left(m_{i}^{\prime}, x_{i}^{\prime}\right) \geqslant\left(m_{i}, x_{i}\right)$ then, for each $s \in S,\left(m_{i}^{\prime}, x_{i}^{\prime}, s\right) P_{i}$ $\left(m_{i}, x_{i}, s\right)$. Assume also that for each $s \in S$, preferences over the set $\mathbb{R}^{2} \times\{s\}$ are strictly convex.

For this section, we extend the meaning of zero commodity indifference to account for the presence of money, but we do not alter its name.

Zero Commodity Indifference: For each quantity $m_{i} \in \mathbb{R}$, each pair $s, t \in S,\left(\left(m_{i}, 0\right), s\right) I_{i}$ $\left(\left(m_{i}, 0\right), t\right)$.

In what follows, assume preferences satisfy zero commodity indifference.

Given prices $p$, agent $i$ 's budget set is $B_{i}(p):=\left\{\left(m_{i}, x_{i}, s\right): p_{s} x_{i}+m_{i} \leq w_{i}\right\}$. Define vector function $z_{i}^{*}$ so that for each $s \in S, z_{i s}^{*}\left(R_{i} ; p\right) \in \mathbb{R}^{2}$ is the unique favourite element of $R_{i}$ from the set $B_{i s}(p):=B_{i}(p) \cap\left(\mathbb{R}^{2} \times\{s\}\right) .{ }^{10}$ Let $z_{i s}^{*}\left(R_{i} ; p\right):=\left(m_{i s}^{*}\left(R_{i} ; p\right), x_{i s}^{*}\left(R_{i} ; p\right)\right)$, where $m_{i s}^{*}$ is the optimal choice of money and $x_{i s}^{*}$ the optimal choice of resource quantity. The demand correspondence is therefore

$$
D_{i}\left(R_{i} ; p\right):=\left\{\begin{array}{c}
x_{i}=x_{i s}^{*}\left(R_{i} ; p\right)>0, m_{i}=m_{i s}^{*}\left(R_{i} ; p\right), \\
\forall t \in S,\left(m_{i}, x_{i}, s\right) R_{i}\left(m_{i t}^{*}\left(R_{i} ; p\right), x_{i t}^{*}\left(R_{i} ; p\right), t\right)
\end{array}\right\} .
$$

Note that since endowments are now personalized, $D_{i}$ must be indexed by agent. As before, we let $D_{i S}$ denote the projection of the demand correspondence onto $S$.

If $(p, z, \alpha)$ is an equilibrium for economy $R$, then for each $i \in N, z_{i}=z_{i \alpha(i)}^{*}\left(R_{i} ; p\right)$. Thus, the pair $(p, \alpha)$ is sufficient to identify an equilibrium.

An economy can be summarized by the list $(N, S, R, w, e)$, where $w \in \mathbb{R}^{N}$. For the following theorem, the elements $(R, w, e)$ all remain constant. Thus, we summarize an economy by the pair $(N, S)$. A typical reduced economy is a list $\left(N^{\prime}, S^{\prime},\left(R_{i}\right)_{i \in N^{\prime}},\left(w_{i}\right)_{i \in N^{\prime}},\left(e_{s}\right)_{s \in S^{\prime}}\right)$, which we denote simply by $\left(N^{\prime}, S^{\prime}\right)$. With some abuse of notation, we write $(p \wedge q) \in \mathbb{P}\left(N^{\prime}, S^{\prime}\right)$ if the list $\left(p_{s} \wedge q_{s}\right)_{s \in S^{\prime}}$ is an equilibrium price for economy $\left(N^{\prime}, S^{\prime}\right)$.

Theorem 7. For each $R \in \mathcal{R}^{N}$, if $\mathbb{P}(R)$ contains a finite price vector, then $(\mathbb{P}(R), \wedge)$ is a lower semi-lattice.

Proof. Let $R \in \mathcal{R}^{N}$. Let $p$ and $q \in \mathbb{P}(R)$. Assume $p, q<(\infty, \ldots, \infty)$. Let $\alpha_{p}$ and $\alpha_{q}$ be site assignments such that $\left(p, \alpha_{p}\right)$ and $\left(q, \alpha_{q}\right)$ are equilibria for $(N, S)$. If $i \in N$ has $D_{s}\left(R_{i}, p \wedge q\right)=\emptyset$,

\footnotetext{
$\overline{{ }^{10} \text { While } B_{i s}(p)} \subseteq \mathbb{R}^{2} \times\{s\}$, it embeds naturally in $\mathbb{R}^{2}$, and so with some abuse of notation, we view it as a subset of $\mathbb{R}^{2}$.
} 
then we give him bundle $\left(\left(w_{i}, 0\right), \emptyset\right)$; he has no effect on the existence of an equilibrium for $p \wedge q$. Thus, it is without loss of generality to assume that, for each $i \in N, D_{s}\left(R_{i}, p \wedge q\right) \neq \emptyset$.

Partition $S$ and $N$ as follows

$$
\begin{array}{ll}
S^{p}:=\left\{s: p_{s}<q_{s}\right\} & N^{p}:=\left\{i: D_{S}\left(R_{i}, p \wedge q\right) \subseteq S_{p}\right\} \\
S^{q}:=\left\{s: q_{s}<p_{s}\right\} & N^{p}:=\left\{i: D_{S}\left(R_{i}, p \wedge q\right) \subseteq S_{q}\right\} \\
S^{=}:=\left\{s: p_{s}=q_{s}\right\} & N^{=}:=N \backslash\left(N_{p} \cup N_{q}\right) .
\end{array}
$$

We will repeatedly use the following fact: for each $s \in S$ and each pair of price vectors $p^{\prime}, q^{\prime} \in \mathbb{R}^{S}$, if $p_{s}^{\prime}=q_{s}^{\prime}$ then $z_{i s}^{*}\left(R_{i} ; p^{\prime}\right)=z_{i s}^{*}\left(R_{i} ; q^{\prime}\right)$.

Claim 2. $(p \wedge q) \in \mathbb{P}\left(N \backslash N^{p}, S \backslash S^{p}\right)$ and $(p \wedge q) \in \mathbb{P}\left(N \backslash N^{q}, S \backslash S^{q}\right)$.

Proof. We show the proof of the first statement. The proof of the second is symmetric.

Let $\hat{\alpha}:=\left.\alpha_{q}\right|_{N \backslash N_{p}}$, the restriction of $\alpha_{q}$ to $N \backslash N^{p}$. Let $i \in N \backslash N^{p}$. There is a site $t \in S \backslash S^{p}$ such that $t \in D_{S}\left(R_{i}, p \wedge q\right)$. Let $s \in S^{p}$.

$$
\begin{aligned}
\left(z_{i t}^{*}\left(R_{i} ; q\right), t\right)= & \left(z_{i t}^{*}\left(R_{i} ; p \wedge q\right), t\right) . \\
R_{i} & \left(z_{i s}^{*}\left(R_{i} ; p \wedge q\right), s\right) \\
R_{i} & \left(z_{i s}^{*}\left(R_{i} ; q\right), s\right) .
\end{aligned}
$$

Assume that the relation on line 5 is $I_{i}$. Since $s \in S^{p}, p_{s}<q_{s}$ and $B_{i s}(p \wedge q)=B_{i s}(p) \supsetneq$ $B_{i s}(q)$. Also, $\left(\mathbb{R}_{+}^{2} \backslash B_{i s}(p)\right) \cap B_{i s}(q)=\left\{\left(w_{i}, 0\right)\right\}$. Thus, $z_{i s}^{*}\left(R_{i} ; p \wedge q\right)=z_{i s}^{*}\left(R_{i} ; p\right)=\left(w_{i}, 0\right)$, and by zero commodity indifference, $\left(z_{i s}^{*}\left(R_{i} ; q\right), s\right) I_{i}\left(\left(w_{i}, 0\right), t\right)$. Then, since $x_{i t}^{*}\left(R_{i} ; q\right)>0$, (as $t \in$ $\left.D_{S}\left(R_{i}, p \wedge q\right)\right)$, we have $\left(z_{i t}^{*}\left(R_{i} ; q\right), t\right) P_{i}\left(\left(w_{i}, 0\right), t\right)$ and therefore the relation on line 4 is $P_{i}$. We conclude that at least one of the relations in lines 4 and 5 is $P_{i}$, and therefore, $\left(z_{i t}^{*}\left(R_{i} ; q\right), t\right) P_{i}$ $\left(z_{i s}^{*}\left(R_{i} ; q\right), s\right)$. It follows that $\alpha_{q}\left(N \backslash N^{p}\right) \subseteq S \backslash S^{p}$, which is of course necessary for $\hat{\alpha}$ to be a site assignment for $\left(N \backslash N^{p}, S \backslash S^{p}\right)$.

Now let $t \in S \backslash S^{p}$ be arbitrary. Since $\left(q, \alpha_{q}\right)$ is an equilibrium for $(N, S)$, for each $i \in N \backslash N^{p}$,

$$
\begin{aligned}
\left(z_{i \hat{\alpha}_{q}(i)}^{*}\left(R_{i} ; p \wedge q\right), \hat{\alpha}(i)\right)= & \left(z_{i \hat{\alpha}_{q}(i)}^{*}\left(R_{i} ; q\right), \hat{\alpha}(i)\right) \\
& R_{i} \quad\left(z_{i t}^{*}\left(R_{i} ; q\right), t\right) \\
= & \left(z_{i t}^{*}\left(R_{i} ; p \wedge q\right), t\right) .
\end{aligned}
$$

Thus $\hat{\alpha}(i)$ is an optimal choice from $S \backslash S^{p}$ given prices $\left(p_{s} \wedge q_{s}\right)_{s \in S \backslash S^{p}}$. 
It remains to show that price $p \wedge q$ with assignment $\hat{\alpha}$ yields a feasible allocation for $\left(N \backslash N^{p}, S \backslash S^{p}\right)$. Since $\left(q, \alpha_{q}\right)$ is an equilibrium, for each $s \in S, \sum_{i \in \alpha_{q}^{-1}(s)} x_{i s}^{*}\left(R_{i} ; q\right) \leq e_{s}$. For each $s \in S \backslash S^{p}$, since $p_{s} \wedge q_{s}=q_{s}$

$$
\sum_{i \in \hat{\alpha}^{-1}(s)} x_{i s}^{*}\left(R_{i} ; p \wedge q\right) \leq \sum_{i \in \alpha_{q}^{-1}(s)} x_{i s}^{*}\left(R_{i} ; p \wedge q\right)=\sum_{i \in \alpha_{q}^{-1}(s)} x_{i s}^{*}\left(R_{i} ; q\right) \leq e_{s} .
$$

Thus the Endowment Constraint is satisfied. Clearly $\left|\hat{\alpha}^{-1}(s)\right| \leq\left|\alpha_{q}^{-1}(s)\right|$, so the Capacity Constraint is satisfied as well.

Claim 3. If $(p \wedge q) \in \mathbb{P}\left(N \backslash N^{q}, S \backslash S^{q}\right)$ and $(p \wedge q) \in \mathbb{P}\left(N \backslash N^{p}, S \backslash S^{p}\right)$, then $(p \wedge q) \in \mathbb{P}(N, S)$.

Proof. Let $\hat{\alpha}$ be a site-assignment such that the list $\hat{z}:=\left(z_{i \hat{\alpha}(i)}^{*}\left(R_{i} ; p \wedge q\right)\right)_{i \in N \backslash N_{p}}$ yields an equilibrium, with price vector $p \wedge q$, for economy $\left(N \backslash N^{p}, S \backslash S^{p}\right)$. Similarly, $\tilde{\alpha}$ be a site-assignment such that the analogous list, $\tilde{z}:=\left(z_{i \tilde{\alpha}(i)}^{*}\left(R_{i} ; p \wedge q\right)\right)_{i \in N \backslash N_{q}}$, yields an equilibrium, with price vector $p \wedge q$, for economy $\left(N \backslash N^{q}, S \backslash S^{q}\right)$. Now define $(z, \alpha)$ as follows:

$$
\alpha^{-1}(s):=\left\{\begin{array}{ll}
\hat{\alpha}^{-1}(s) & s \in S_{q} \\
\tilde{\alpha}^{-1}(s) & s \in S \backslash S_{q}
\end{array} \quad z_{i}:=x_{i \alpha(i)}^{*}\left(R_{i} ; p \wedge q\right) .\right.
$$

Note that if $\alpha(i)=\hat{\alpha}(i)$ then $z_{i}=\hat{z}_{i}$, and if $\alpha(i)=\tilde{a}(i)$ then $z_{i}=\tilde{z}_{i}$. We first verify that $(z, \alpha)$ gives each agent an optimal choice given prices $p \wedge q$. Let $i \in N_{q}$. Then $\alpha(i)=\hat{\alpha}(i)$. Since $(p \wedge q, \hat{z}, \hat{\alpha})$ is an equilibrium for $\left(N \backslash N^{p}, S \backslash S^{p}\right)$, and since $D_{i S}\left(R_{i}, p \wedge q\right) \cap S^{p}=\emptyset$, we have $\left(\hat{z}_{i}, \hat{\alpha}(i)\right) \in D\left(R_{i}, p \wedge q\right)$. If $i \in N_{p}$, then $\alpha(i)=\tilde{\alpha}(i)$ and the argument is symmetric with the previous case. Finally, assume $i \in N^{=}$. Then there exist $\hat{s} \in S \backslash S^{p}$ and $\tilde{s} \in S \backslash S^{q}$, not necessarily distinct, such that $\{\hat{s}, \tilde{s}\} \subseteq D_{S}\left(R_{i}, p \wedge q\right)$. It follows that

$$
\begin{aligned}
\left(\hat{z}_{i}, \hat{\alpha}(i)\right) & =\left(z_{i \hat{\alpha}(i)}^{*}\left(R_{i} ; p \wedge q\right), \hat{\alpha}(i)\right) \\
& I_{i}\left(z_{i \hat{s}}^{*}\left(R_{i} ; p \wedge q\right), \hat{s}\right) \\
& I_{i}\left(z_{i \tilde{s}}^{*}\left(R_{i} ; p \wedge q\right), \tilde{s}\right) \\
& I_{i}\left(z_{i \tilde{\alpha}(i)}^{*}\left(R_{i} ; p \wedge q\right), \tilde{\alpha}(i)\right)=\left(\tilde{z}_{i}, \tilde{\alpha}(i)\right) .
\end{aligned}
$$

Thus, $\left(z_{i}, \alpha(i)\right) \in D\left(R_{i}, p \wedge q\right)$.

Feasibility of $(z, \alpha)$ follows immediately from its construction.

Claims 2 and 3 give the result. 
For the application of graduate school admissions, we assume that agents gain no welfare from the consumption of the numeraire. Thus, preferences over $\left\{\left(m_{i}, x_{i}, s_{i}\right):\left(m_{i}, x_{i}\right) \in \mathbb{R}^{2}\right\}$ are not strictly convex. However, it remains the case that $x_{i s}^{*}\left(R_{i} ; p\right)$ is single-valued, and this is enough to make the proof work.

Continuity of preferences implies the closedness of $(\mathbb{P}(R), \wedge)$. Since $(\mathbb{P}(R), \wedge)$ is also bounded below by 0 , there is a unique minimal price vector $p^{*}(R)$ for economy $R$. The correspondence of equilibria that result by using $p^{*}(R)$ as the price for economy $R$ is essentially-single-valued. Thus, we may define a min-price Walrasian rule for this more general model.

\section{Conclusion}

We have developed a rich and flexible framework for analysing a novel class of problems. If all participating agents are equal in the eyes of the designer, we have characterized the best incentivecompatible allocation rule. By studying the rule that resulted, we were able to define rules for a rich family of problems where agents may be differentiated by their willingness to spend money or preference-irrelevant characteristics. Clearly, we anticipate that the good qualities of the benchmark rule should persist in the extended family of rules, but verifying this is beyond the scope of this paper.

\section{Appendix A. Proofs For Section 3}

For each $p \in \overline{\mathbb{R}}_{+}^{S}$, each $s \in S$, let $\llbracket p, s \rrbracket$ denote the bundle $\left(\frac{w}{p_{s}}, s\right) \in \mathbb{R}_{+} \times S$. For site assignment $\alpha \in S^{N}$, let $\llbracket p, \alpha \rrbracket$ denote the allocation such that each $i \in N$ consumes $\llbracket p, \alpha(i) \rrbracket$. Allocation $\llbracket p, \alpha \rrbracket$ need not be feasible and each agent's bundle need not be an optimal choice from the $p$ budget set, but it is clear that we may restrict our attention to allocations of this form when searching for equilibria.

A.1. Some topological properties. Fix the consumption space

$$
Z:=\left\{\left(x_{0}, s_{0}\right) \in \mathbb{R} \times S: 0 \leq x_{0} \leq e_{s_{0}}\right\} .
$$

Endow $Z$ with the metric $\rho$ defined for each pair $\left\{\left(x_{0}, s_{0}\right),\left(y_{0}, t_{0}\right)\right\}$ by setting

$$
\rho\left[\left(x_{0}, s_{0}\right),\left(y_{0}, t_{0}\right)\right]:= \begin{cases}\left|x_{0}-y_{0}\right| & s_{0}=t_{0} \\ 2 \max _{s \in S} e_{s} & s_{0} \neq t_{0} .\end{cases}
$$


Let the metric on $Z \times Z$ be given, for each pair $\left\{\left(z^{1}, z^{2}\right),\left(\hat{z}^{1}, \hat{z}^{2}\right)\right\}$, by $\max \left\{\rho\left(z^{1}, \hat{z}^{1}\right), \rho\left(z^{2}, \hat{z}^{2}\right)\right\}$. A preference relation is a closed subset $R \subset Z \times Z$. Since $Z \times Z$ is compact, the Hausdorff distance $H^{\Delta}$ is a metric for the space of preference relations $\mathcal{R}$. In fact, the topology induced by $H^{\Delta}$ is precisely the topology of closed-convergence. See Hildenbrand (1974). Endow $\mathcal{R}^{N}$ with the product topology.

Lemma 3. Let $p^{n} \rightarrow p \in \mathbb{R}_{++}^{S}$ and $R_{i}^{n} \rightarrow R_{i}$. There exists $\bar{n} \in \mathbb{N}$ such that for each $n \geq \bar{n}$, $D_{S}\left(R_{i}^{n}, p^{n}\right) \subseteq D_{S}\left(R_{i}, p\right)$.

Proof. Let $s_{0} \notin D_{S}\left(R_{i}, p\right)$. Let

$$
\varepsilon:=\max \left\{\frac{w}{p_{t}}-x_{0}:\left(x_{0}, t\right) I_{i}\left(\frac{w}{p_{s_{0}}}, s_{0}\right)\right\} .
$$

Since $s_{0} \notin D\left(R_{i}, p\right), \varepsilon>0$. Assume $\left(z_{0}, r_{0}\right) I_{i}\left(w p_{s_{0}}^{-1}, s_{0}\right)$ satisfies $w p_{r_{0}}^{-1}-z_{0}=\varepsilon$. For each $n \in \mathbb{N}$, let $\left(z(n), r_{0}\right) I_{i}\left(w\left[p_{s_{0}}^{n}\right]^{-1}, s_{0}\right)$. Since $p^{n} \rightarrow p$ and $p$ has no zero components, $w\left[p_{r_{0}}^{n}\right]^{-1} \rightarrow$ $w p_{r_{0}}^{-1}$. Therefore, since preferences are continuous, there is $n^{1} \in \mathbb{N}$ such that for each $n>n^{1}$, $w\left[p_{r_{0}}^{n}\right]^{-1}-z(n)>\varepsilon / 2$. For each $n$, let $x(n)$ satisfy $\left(x(n), r_{0}\right) I_{i}^{n}\left(w\left[p_{s_{0}}^{n}\right]^{-1}, s_{0}\right)$. The topology on $\overline{\mathcal{R}}$ implies there is an $n^{2} \in \mathbb{N}$ such that for each $n>n^{2},|z(n)-x(n)|<\varepsilon / 2$. Let $\bar{n}_{s_{0}}=\max \left\{n^{1}, n^{2}\right\}$. Then for each $n>\bar{n}_{s_{0}}$.

$$
\begin{aligned}
\frac{w}{p_{r_{0}}^{n}}-x(n) & \geq \frac{w}{p_{r_{0}}^{n}}-z(n)+z(n)-x(n) \\
& >\frac{\varepsilon}{2}+z(n)-x(n)>0 .
\end{aligned}
$$

Therefore, for each $n \geq \bar{n}_{s_{0}}, s_{0} \notin D_{S}\left(R_{i}^{n}, p^{n}\right)$.

Let $\bar{n}:=\max \left\{\bar{n}_{s_{0}}: s_{0} \notin D_{S}\left(R_{i}, p\right)\right\}$. We have shown that for each $n>\bar{n}, S \backslash D_{S}\left(R_{i}, p\right) \subseteq$ $S \backslash D_{S}\left(R_{i}^{n}, p^{n}\right)$ and the result follows.

We define the limit inferior of a sequence $x^{n} \in \mathbb{R}^{K}$ component-wise: for each $k$, let $\underline{x}_{k}:=$ $\liminf x_{k}^{n}$. Then define $\liminf x^{n}:=\underline{x}$. Note that

$$
\liminf x^{n}=\lim _{n \rightarrow \infty}\left[\inf _{\leq}\left\{x^{\tilde{n}}: \tilde{n} \geq n\right\}\right],
$$

since the interior infimum can be found component-wise and still results in a non-decreasing sequence in the vector order. The limit superior is symmetric.

Lemma 4. $p^{*}$ is lower semi-continuous. 
Proof. Let $R^{n} \rightarrow R$. Let $p:=\liminf p^{*}\left(R^{n}\right)$. Let $s \in S$. There is a sub-sequence $R^{\sigma(n)}$ such that $\lim p_{s}^{*}\left(R^{\sigma(n)}\right)=p_{s}$. Let $p^{1}:=\liminf p^{*}\left(R^{\sigma(n)}\right)$. For $t \neq s$, there is a further sub-sequence $R^{\tau(n)}$ such that $\lim p_{t}^{*}\left(R^{\tau(n)}\right)=p_{t}^{1}$. By repeating the process, we find a sub-sequence $R^{v(n)}$ and a price vector $p^{s}$ such that $\lim p^{*}\left(R^{v(n)}\right)=p^{s}$ and $p_{s}^{s}=p_{s}$. Since $S^{N}$ is finite, there is a site assignment $\alpha \in S^{N}$ and a further sub-sequence $R^{\tilde{\gamma}(n)}$ such that for each $n, \alpha \in \mathcal{A}^{*}\left(R^{\tilde{\gamma}(n)}\right)$. By Lemma 3, for each $i \in N$, $\alpha(i) \in D_{S}\left(R_{i}, p^{s}\right)$. Therefore, $\llbracket p^{s}, \alpha \rrbracket$ is an equilibrium for $R$ and $p^{s} \in \mathbb{P}(R)$. Since $s$ was arbitrary, we note that, by the lower semi-lattice property of $\mathbb{P}(R)$ (Theorem 1) $p=\bigwedge_{s \in S} p^{s} \in \mathbb{P}(R)$. Finally, minimality of $p^{*}$ yields

$$
p^{*}(R) \leq p=\liminf p^{*}\left(R^{n}\right)
$$

A.2. Blocking via Indifference. To facilitate discussion of blocking via indifference, it is helpful to borrow language from graph theory. Given economy $R \in \mathcal{R}^{N}$, prices $p \in \mathbb{R}^{S}$, and site-assignment $\alpha \in \mathcal{A}^{*}(R)$, we shall construct a directed graph with labelled arcs, denoted $\Gamma(R ; p, \alpha)$. The vertex set is $S$ and the label set is $N$. Since these remain constant, reference to them is suppressed, and so, with abuse of notation, $\Gamma(R ; p, \alpha)$ refers to the set of arcs. If agent $i \in N$ is assigned $\alpha(i)=s$, and $\llbracket p^{*}(R), t \rrbracket R_{i} \llbracket p^{*}(R), s \rrbracket$, where $t \neq s$, then $(s, t, i) \in \Gamma(R ; p, \alpha)$. In the visual representation of the graph, there is an arc from $s$ to $t$ with label $i$. We may write this $s \stackrel{i}{\rightarrow} t \in \Gamma(R ; p, \alpha)$, and we may suppress the arc label where it is not important. Note that if $(p, x, \alpha)$ is an equilibrium, then each arc in $\Gamma(R ; p, \alpha)$ represents an indifference relation. A generic path from $r^{0}$ to $r^{m}$ is denoted $r^{0} \leadsto r^{m}$. A path $r^{0} \rightsquigarrow r^{m}$ is maximal in the graph $\Gamma$ if there is no $\operatorname{arc}\left(r^{\prime}, r^{0}\right) \in \Gamma$ and no arc $\left(r^{m}, r^{\prime \prime}\right) \in \Gamma$.

Theorem 2 is the union of Lemmas 5, 7, and 8.

Lemma 5. Let $R \in \overline{\mathcal{R}}^{N}$ and $(x, \alpha) \in F^{*}(R)$ be supported by prices $p$. Assume that $s$ is not exhausted at equilibrium $(p, x, \alpha)$. Then $s$ is blocked via indifference by a site that is exhausted at $(p, x, \alpha)$.

Proof. Assume $s^{*} \in S$ is not exhausted at $(p, x, \alpha)$. Let $\Gamma^{\prime} \subseteq \Gamma\left(R ; p^{*}(R), \alpha\right)$ be the graph composed of all paths ending in $s^{*}$. That is, $r \stackrel{i}{\rightarrow} t \in \Gamma^{\prime}$ if and only if there is a path $s \rightsquigarrow s^{*} \subseteq \Gamma\left(R ; p^{*}(R), \alpha\right)$ such that $r \stackrel{i}{\rightarrow} t \in s \rightsquigarrow s^{*}$. Let $V \subseteq S$ be the sites adjacent to some arc in $\Gamma^{\prime}$. By hypothesis, no element of $V$ is exhausted at $\llbracket p^{*}(R), \alpha \rrbracket$. By construction, $V$ is a source of $\Gamma\left(R ; p^{*}(R), \alpha\right)$ : there is no arc $r \rightarrow t \in \Gamma\left(R ; p^{*}(R), \alpha\right)$ with $r \in S \backslash V$ and $t \in V$. To arrive at a contradiction, assume that for each $t \in V, t$ is not exhausted at $(p, x, \alpha)$. By continuity of preferences, there is $\varepsilon>0$ and a 
price vector $p^{\varepsilon} \in \mathbb{R}^{S}$, given for each $s \in S$ by

$$
p_{s}^{\varepsilon}:= \begin{cases}p_{s}^{*}(R)-\varepsilon & s \in V \\ p_{s}^{*}(R) & s \notin V,\end{cases}
$$

such that $\llbracket p^{\varepsilon}, \alpha \rrbracket$ is feasible and $V$ is also a source of $\Gamma\left(R ; p^{\varepsilon}, \alpha\right)$. Note that $\llbracket p^{\varepsilon}, \alpha \rrbracket$ is not necessarily an equilibrium; if it were, the proof would be finished here.

Focus attention on the sub-economy given by sites $V$ and agents $N^{\star}:=\alpha^{-1}(V)$. Denote this sub-economy $\mathcal{E}$. We transform $\mathcal{E}$ as follows. Let $\underline{c} \in \mathbb{N}^{V}$ satisfy, for each $s \in V, \underline{c}_{s}=\left|\alpha^{-1}(s)\right|$. Construct the set $V^{\star}$ from $V$ by having each site $s$ exist as $\underline{c}_{s}$ identical copies. Copies of $s$ are denoted $s^{a}, s^{b}$, etc. Agent $i$ 's preferences $R_{i}^{\star}$ over $\mathbb{R} \times V^{\star}$ are defined in the natural way: for each $x \in \mathbb{R},\left\{s^{a}, s^{b}\right\} \subseteq\{s\}^{\star}$ implies $\left(x, s^{a}\right) I_{i}^{\star}\left(x, s^{b}\right)$ and otherwise $R_{i}^{\star}$ respects $R_{i}$. We define an endowment vector $e^{\star}$ for each $s^{a} \in\{s\}^{\star}$ as

$$
e_{s^{a}}^{\star}=\frac{w}{p_{s}^{\varepsilon}}
$$

Finally define capacity vector $c^{\star} \in \mathbb{N}^{V^{\star}}$ by $c^{\star}=(1,1, \ldots, 1)$. Let $\mathcal{E}^{\star}$ be the economy consisting of sites $V^{\star}$ with capacities $c^{\star}$, endowments $e^{\star}$, and agents $N^{\star}$ having preferences $R^{\star}$. This economy admits an equilibrium $\llbracket p^{\star}, \alpha^{\star} \rrbracket$ by adapting $\llbracket p, \alpha \rrbracket$ in the obvious way. Then by Lemma 3 in Demange and Gale (1985), it admits an equilibrium $\llbracket q^{\star}, \beta^{\star} \rrbracket$ such that there is a site $r$, having a copy $r^{a}$, such that

$$
\frac{w}{q_{r^{a}}^{\star}}=e_{r^{a}}^{\star}
$$

implying that $q_{r^{a}}^{\star}=p_{r^{\varepsilon}}^{\varepsilon}{ }^{11}$ Moreover the lattice structure allows us to assume $q^{\star} \leq p^{\star}$.

Let $\left\{t^{a}, t^{b}\right\} \subseteq\{t\}^{\star}$. By the construction of $R^{\star}, q_{t^{a}}^{\star}=q_{t^{b}}^{\star}$. Thus, $q_{t}:=q_{t^{a}}^{\star}$ is well-defined. Define $\beta$ for each site $s \in V$ by $\beta^{-1}(s):=\cup_{s^{a} \in\{\}^{\star}}\left\{\beta^{\star-1}\left(s^{a}\right)\right\}$. We have constructed an equilibrium $\llbracket q, \beta \rrbracket$ for $\mathcal{E}$ such that for each $s \in V, q_{s} \leq p_{s}$. Moreover,

$$
q_{r}=p_{r}^{\varepsilon}<p_{r}
$$

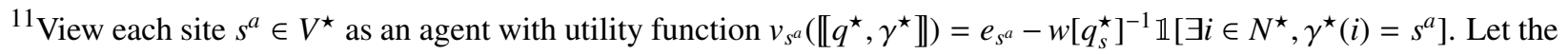
outside option of each $s^{a}$ be 0 . We have a model of one to one matching where stability coincides with equilibrium. Our $V^{\star}$ corresponds to $Q$ in Demange and Gale (1985), and our $N^{\star}$ corresponds to $P$. By construction, $\left|V^{\star}\right| \geq\left|N^{\star}\right|$.
} 
Now we map back to the original economy, with sites $S$ and agents $N$. Extend $q$ to each $t \in S \backslash V$ by setting $q_{t}:=\infty$. Let

$$
\gamma(j):= \begin{cases}\alpha(j) & j \in N \backslash N^{\star} \\ \beta(j) & j \in N^{\star} .\end{cases}
$$

By construction $\llbracket p \wedge q, \gamma \rrbracket$ is an equilibrium for $R$, contradicting the minimality of $p^{*}(R)$.

Given $\Gamma(R ; p, \alpha)$ and a path $r^{0} \leadsto r^{m} \subseteq \Gamma(R ; p, \alpha)$, site-assignment $\alpha^{\prime} \in S^{N}$ is the path shift of $\alpha$ via $r^{0} \leadsto r^{m}$ if, for each $i \in N$,

$$
\alpha^{\prime}(i):= \begin{cases}r^{l+1} & \left(r^{l}, r^{l+1}, i\right) \in r^{0} \rightsquigarrow r^{m} \\ \alpha(i) & \text { otherwise. }\end{cases}
$$

It is easy to find the relation between $\Gamma(R ; p, \alpha)$ and $\Gamma\left(R ; p, \alpha^{\prime}\right)$ : the $\operatorname{arcs}$ in $r^{0} \rightsquigarrow r^{m}$ are reversed and all else is the same. Formally, the mapping $\psi: \Gamma(R ; p, \alpha) \rightarrow \Gamma\left(R ; p, \alpha^{\prime}\right)$, defined for each $\left(r^{\prime}, r^{\prime \prime}, k\right) \in \Gamma(R ; p, \alpha)$ by

$$
\psi\left(r^{\prime}, r^{\prime \prime}, k\right):= \begin{cases}\left(r^{l+1}, r^{l}, k\right) & r^{l+1}=r^{\prime \prime} \text { and } r^{l}=r^{\prime} \\ \left(r^{\prime}, r^{\prime \prime}, k\right) & \text { otherwise, }\end{cases}
$$

is a bijection.

Lemma 6. Let $\alpha \in \mathcal{A}^{*}(R)$ and $r \leadsto s \subseteq \Gamma\left(R ; p^{*}(R), \alpha\right)$. Let $\alpha^{\prime}$ be the path shift of $\alpha$ via $r \rightsquigarrow s$ and assume that $\llbracket p^{*}(R), \alpha^{\prime} \rrbracket$ is feasible. Then $\alpha^{\prime} \in \mathcal{A}^{*}(R)$.

Proof. Let $i \in N$. If $\alpha^{\prime}(i)=\alpha(i)$ then clearly $\alpha(i) \in D_{S}\left(R_{i} ; p^{*}(R)\right)$. Otherwise, there is an arc $r^{l} \stackrel{i}{\rightarrow} r^{l+1} \in \Gamma\left(R ; p^{*}(R), \alpha\right)$. Then by definition, $\llbracket p^{*}(R), r^{l+1} \rrbracket R_{i} \llbracket p^{*}(R), r^{l} \rrbracket$. Therefore, since $r^{l}=\alpha(i) \in D_{S}\left(R_{i} ; p^{*}(R)\right)$, it follows that $r^{l+1} \in D_{S}\left(R_{i} ; p^{*}(R)\right)$.

Lemma 7. For each profile $R \in \overline{\mathcal{R}}^{N}$ such that $\mathbb{P}(R) \neq \emptyset$, there is a balanced site assignment $\alpha \in \mathcal{A}^{*}(R)$.

Proof. Without loss of generality, we may normalize the endowment vector to $e=\mathbf{1}$ and the distributed numeraire to $w=1$. In this case, for each $s \in S, \tilde{c}_{s}\left(p^{*}(R)\right)=\left\lfloor p_{s}^{*}(R)\right\rfloor$. Let $R \in \mathcal{R}^{N}$, $p:=p^{*}(R)$, and $\alpha \in \mathcal{A}^{*}(R)$. Let $S^{\prime}:=\left\{s \in S: p_{s} \notin \mathbb{Z}\right.$ and $\left.\left|\alpha^{-1}\right|<c_{s}\right\}$.

Suppose there is $s \in S^{\prime}$ with $\left|\alpha^{-1}(s)\right|<c_{s}(p)-\mathbb{1}\left[c_{s} \geq \tilde{c}_{s}(p)\right]$. By Lemma 5 , there is a path $s^{0} \leadsto s \subseteq \Gamma(R ; p, \alpha)$ such that $s^{0}$ is exhausted at $\llbracket p, \alpha \rrbracket$. Let $\beta$ be the path-shift of $\alpha$ via $s^{0} \leadsto s$. Clearly, $\llbracket p, \beta \rrbracket$ is feasible, so by Lemma $6, \beta \in \mathcal{A}^{*}(R)$. Since site $s^{0}$ is exhausted under $\llbracket p, \alpha \rrbracket$, 
$\left|\alpha^{-1}\left(s^{0}\right)\right|=\tilde{c}_{s^{0}}(p) \leq c_{s^{0}}$. Thus, $\left|\beta^{-1}\left(s^{0}\right)\right|=c_{s^{0}}(p)-1$. For each $t \in S \backslash\left\{s, s^{0}\right\},\left|\beta^{-1}(t)\right|=\left|\alpha^{-1}(t)\right|$. Finally, $\left|\beta^{-1}(s)\right|=\left|\alpha^{-1}(s)\right|+1$.

If $\left|\beta^{-1}(s)\right| \leq c_{s}(p)-1$, we repeat the exercise. In the next iteration, there is $s^{1} \leadsto s \subseteq \Gamma(R ; p, \alpha)$ such that $s^{1} \neq s^{0}$ and $s^{1}$ is exhausted at $\llbracket p, \beta \rrbracket$. Proceeding thus, we generate a list of sites $\left\{s^{0}, s^{1}, \ldots, s^{k}\right\}$ and a site assignment $\gamma \in \mathcal{A}^{*}(R)$ such that for each $s^{l} \in\left\{s^{0}, s^{1}, \ldots, s^{k}\right\}, p_{s^{l}} \in \mathbb{Z}$ and $\left|\gamma^{-1}\left(s^{l}\right)\right|=c_{s^{l}}(p)-1$. Moreover, $\left|\gamma^{-1}(s)\right|=\left|\alpha^{-1}(s)\right|+k+1$. Finally, for each $t \notin\left\{s, s^{0}, \ldots, s^{k}\right\}$, $\left|\gamma^{-1}(t)\right|=\left|\alpha^{-1}(t)\right|$. For $k$ large enough, either $s$ is no longer blocked via indifference by an exhausted site, or $\left|\gamma^{-1}(s)\right|=c_{s}(p)$. The former case is ruled-out by Lemma 5. Thus the latter case is true and the lemma is proved.

Lemma 8. Let $\llbracket p, \alpha \rrbracket$ be a balanced equilibrium such that each site $s \in S$ is blocked via indifference by an exhausted site. Then $\llbracket p, \alpha \rrbracket \in F^{*}(R)$.

Proof. Assume $\llbracket p, \alpha \rrbracket$ is a balanced equilibrium. Assume there are a price $p^{\prime} \leq p$ and a site assignment $\beta \in S^{N}$ such that $\llbracket p^{\prime}, \beta \rrbracket$ is an equilibrium for $R$. Let $S^{-}:=\left\{s \in S: p_{s}^{\prime}<p_{s}\right\}$. Since $\llbracket p, \alpha \rrbracket$ is a balanced allocation, if $s \in S^{-}$with $\left|\alpha^{-1}(s)\right|=c_{s}(p)-1, s$ has endowment divisible value, and therefore $c_{s}\left(p^{\prime}\right) \leq c_{s}(p)-1$. Thus,

$$
\forall s \in S^{-},\left|\alpha^{-1}(s)\right| \geq c_{s}\left(p^{\prime}\right)
$$

If $D_{S}\left(R_{i}, p\right) \cap S^{-} \neq \emptyset$, then clearly $D_{S}\left(R_{i}, p^{\prime}\right) \subseteq S^{-}$. Since each $i \in \alpha^{-1}\left(S^{-}\right)$has $D_{S}\left(R_{i}, p\right) \cap S^{-} \neq$ $\emptyset, \beta^{-1}\left(S^{-}\right) \supseteq \alpha^{-1}\left(S^{-}\right)$. Line 6 further implies that $\left|\beta^{-1}\left(S^{-}\right)\right|=\left|\alpha^{-1}\left(S^{-}\right)\right|$so $\beta^{-1}\left(S^{-}\right)=\alpha^{-1}\left(S^{-}\right)$. It follows that, $\beta^{-1}\left(S \backslash S^{-}\right)=\alpha^{-1}\left(S \backslash S^{-}\right)$. If $D_{S}\left(R_{i}, p^{\prime}\right) \cap\left(S \backslash S^{-}\right) \neq \emptyset$, then clearly $D_{S}\left(R_{i}, p\right) \subseteq S \backslash S^{-}$. Since each $i \in \beta^{-1}\left(S \backslash S^{-}\right)=\alpha^{-1}\left(S \backslash S^{-}\right)$has $D_{S}\left(R_{i}, p^{\prime}\right) \cap\left(S \backslash S^{-}\right) \neq \emptyset$, conclude that, for each $s \in S^{-}, s \in D_{S}\left(R_{j}, p\right)$ if and only if $\alpha(j) \in S^{-}$. Thus, $S^{-}$is a source in the graph $\Gamma(R ; p, \alpha)$. If at $\llbracket p, \alpha \rrbracket$, each site is blocked via indifference by an exhausted site, $S^{-}$must contain a site, $s^{*}$, exhausted at $\llbracket p, \alpha \rrbracket$. This site has endowment divisible value at $p$ and $\left|\alpha^{-1}\left(s^{*}\right)\right|=c_{s^{*}}(p)$, so

$$
\left|\alpha^{-1}\left(s^{*}\right)\right|=c_{s^{*}}(p) \geq c_{s^{*}}\left(p^{\prime}\right)+1 .
$$

Then, since $\left|\beta^{-1}\left(S^{-}\right)\right|=\left|\alpha^{-1}\left(S^{-}\right)\right|$, lines 6 and 7 yield

$$
\left|\beta^{-1}\left(S^{-}\right)\right|=\left|\alpha^{-1}\left(S^{-}\right)\right| \geq c_{s^{*}}\left(p^{\prime}\right)+1+\sum_{s \in S^{-} \backslash s^{*}} c_{s}\left(p^{\prime}\right),
$$

contradicting feasibility. 
A.3. Proof of Lemma 1. We prove Lemma 1 in parts, with Lemma 2 proved in the process. We can simplify notation as follows: for each $d \in \mathbb{R}, R^{d}:=\left(R_{i}^{s, d}, R_{-i}\right)$.

Proof of Property 2. Since $s \notin D_{S}\left(R_{i}, \pi(0)\right)$, there is an open set $U \subset \mathbb{R}$ containing zero such that

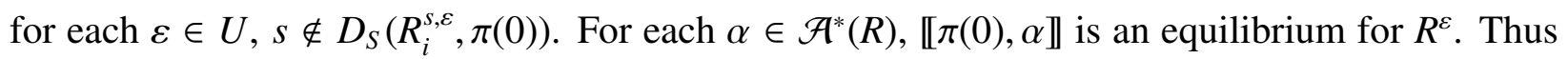
$\pi(\varepsilon) \leq \pi(0)$.

Case 1. There is an open neighbourhood $V$ containing 0 such that for each $\varepsilon \in V, s \notin D_{S}\left(R_{i}^{s, \varepsilon}, \pi(\varepsilon)\right)$. Then if $\llbracket \pi(\varepsilon), \beta \rrbracket$ is an equilibrium for $R^{\varepsilon}, \beta(i) \neq s$. Since $R_{i}$ and $R_{i}^{s, \varepsilon}$ agree on $\mathbb{R} \times S \backslash s$, and since $R_{-i}^{\varepsilon}=R_{-i}, \llbracket \pi(\varepsilon), \beta \rrbracket$ is also an equilibrium for $R$. Thus by minimality, $\pi(0) \leq \pi(\varepsilon)$ and we conclude that $\pi(\varepsilon)=\pi(0)$.

Case 2. There is a sequence $\varepsilon^{n}$ converging to zero such that for each $n \in \mathbb{N}, s \in D_{S}\left(R_{i}^{s, \varepsilon^{n}}, \pi\left(\varepsilon^{n}\right)\right)$. We showed that for each $\varepsilon>0$ sufficiently small, $\pi(0) \geq \pi(\varepsilon)$. Combined with Lemma 4, we may write

$$
\pi(0) \geq \limsup _{n \rightarrow \infty} \pi\left(\varepsilon^{n}\right) \geq \liminf _{n \rightarrow \infty} \pi\left(\varepsilon^{n}\right) \geq \pi(0) .
$$

Therefore $\lim _{n \rightarrow \infty} \pi\left(\varepsilon^{n}\right)=\pi(0)$. By Lemma 3, $s \in D_{S}\left(R_{i}, \pi(0)\right)$, a contradiction.

We have shown that if $s \notin D_{S}\left(R_{i}, p^{*}(R)\right)$, then for $d$ sufficiently small, $\pi(d)=\pi(0)$. For each such $d, \pi(0) \in \mathbb{P}\left(R_{i}^{s, d}, R_{-i}\right)$, and therefore $\pi(0) \geq \pi(d)$. Define $\bar{d}$ as the unique number satisfying $D_{S}\left(R_{i}^{s, \bar{d}_{i}}, p^{*}(R)\right)=D_{S}\left(R_{i}, p^{*}(R)\right) \cup\{s\}$. Let $d^{n}$ be an increasing sequence converging to $\bar{d}$. Then

$$
\pi(0) \geq \lim \sup \pi\left(d^{n}\right) \geq \lim \inf \pi\left(d^{n}\right) \geq \pi(0),
$$

where the final inequality is from the lower semi-continuity of $p^{*}$.

Lemma 9. Assume $\alpha(i)=s$. Then for each $d>0$, each $\beta \in \mathcal{A}^{*}\left(R^{d}\right), \beta(i)=s$.

Proof. Suppose not: there exist $d>0$ and a site assignment $\beta \in \mathcal{A}^{*}\left(R^{d}\right)$ such that $\beta(i)=$ $t \neq s$. Note that $\llbracket p^{*}(R), \alpha \rrbracket$ is an equilibrium for $R^{d}$. Therefore, $p^{*}\left(R^{d}\right) \leq p^{*}(R)$. Since $t \notin$ $D_{S}\left(R_{i}^{s, d}, p^{*}(R)\right)$, it follows by monotonicity of preferences that $p_{t}^{*}\left(R^{d}\right)<p_{t}^{*}(R)$. Since $R_{i}$ is a negative $s$-translation of $R_{i}^{s, d}, \llbracket p^{*}\left(R^{d}\right), \beta \rrbracket$ is an equilibrium for $R$, a contradiction.

Proof of Lemma 2. By Property 2, if $s_{i} \notin D_{S}\left(R_{i}, p^{*}(R)\right)$, then there exists $\bar{d}_{i}$ such that $p^{*}\left(R_{i}^{s_{i}, \bar{d}_{i}}, R_{-i}\right)=$ $p^{*}(R)$ and $s_{i} \in D_{S}\left(R_{i}^{s_{i}, \bar{d}_{i}}, p^{*}(R)\right)$. The same holds for each $i \in N^{\prime}: p^{*}\left(\left(R_{j}^{s_{j}, \bar{d}_{j}}\right)_{i \in N^{\prime}}, R_{N \backslash N^{\prime}}\right)=p^{*}(R)$.

Thus we assume without loss of generality that $R=\left(\left(R_{j}^{s_{j}, \bar{d}_{j}}\right)_{i \in N^{\prime}}, R_{N \backslash N^{\prime}}\right)$. 
Assume $\alpha \in \mathcal{A}^{*}(R)$ is a balanced site-assignment. Let $\bar{R}:=\left(\left(R_{j}^{s_{j}, d}\right)_{j \in N^{\prime}}, R_{N \backslash N^{\prime}}\right), \hat{S}:=\{t \in S:$ $\left.p_{t}^{*}\left(R^{d}\right)<p_{t}^{*}(R)\right\}$, and $\hat{N}:=\left\{i \in N: F^{*}\left(R^{d}\right) P_{i} F^{*}(R)\right\}$. It is clear that if $t \in \hat{S}$ then $\alpha^{-1}(t) \subseteq \hat{N}$. Therefore

$$
\alpha^{-1}(\hat{S}) \subseteq \hat{N}
$$

If $t \in \hat{S}$ has endowment divisible value at $p^{*}(R)$, then $\tilde{c}_{t}\left(p^{*}(\bar{R})\right) \leq \tilde{c}_{t}\left(p^{*}(R)\right)-1$. Since $\alpha$ is balanced, $\min \left\{c_{t}, \tilde{c}_{t}\left(p^{*}(R)\right)-1\right\} \leq\left|\alpha^{-1}(t)\right|$. Thus if $\min \left\{c_{t}, \tilde{c}_{t}\left(p^{*}(R)\right)-1\right\}=\tilde{c}_{t}\left(p^{*}(R)\right)-1$,

$$
c_{t}\left(p^{*}(\bar{R})\right) \leq \tilde{c}_{t}\left(p^{*}(\bar{R})\right) \leq \tilde{c}_{t}\left(p^{*}(R)\right)-1 \leq\left|\alpha^{-1}(t)\right| .
$$

Otherwise, $\min \left\{c_{t}, \tilde{c}_{t}\left(p^{*}(R)\right)-1\right\}=c_{t}$ and we have

$$
c_{t}\left(p^{*}(\bar{R})\right) \leq c_{t} \leq\left|\alpha^{-1}(t)\right| .
$$

Therefore $c_{t}\left(p^{*}(\bar{R})\right) \leq\left|\alpha^{-1}(t)\right|$. If $t \in \hat{S}$ does not have endowment divisible value, $c_{t}\left(p^{*}(\bar{R})\right) \leq$ $c_{t}\left(p^{*}(R)\right)=\left|\alpha^{-1}(t)\right|$. In sum,

$$
\forall t \in \hat{S},\left|\alpha^{-1}(t)\right| \geq c_{t}\left(p^{*}(\bar{R})\right)
$$

To arrive at a contradiction, assume that for each $r \in\left(s_{j}\right)_{j \in N^{\prime}}, p_{r}^{*}(\bar{R})<p_{r}^{*}(R)$. Clearly $N^{\prime} \subseteq \hat{N}$. We claim that for each $k \in \hat{N}, D_{S}\left(\bar{R}_{k}, p^{*}(\bar{R})\right) \subseteq \hat{S}$. By construction, for $k \in N^{\prime}, D_{S}\left(\bar{R}_{k}, p(\bar{R})\right) \subseteq$ $\left(s_{j}\right)_{j \in N^{\prime}} \subseteq \hat{S}$. Let $k \in \hat{N} \backslash N^{\prime}$. Since $\bar{R}_{k}=R_{k}$ and preferences are increasing, $F^{*}(\bar{R}) P_{k} F^{*}(R)$ implies the claim directly. Let $\beta \in \mathcal{A}^{*}(\bar{R})$. What we have just shown implies $\hat{N} \subseteq \beta^{-1}(\hat{S})$. By 9 ,

$$
|\hat{N}| \leq\left|\beta^{-1}(\hat{S})\right| \leq \sum_{r \in \hat{S}} c_{r}\left(p^{*}(\bar{R})\right) \leq \sum_{r \in \hat{S}}\left|\alpha^{-1}(r)\right|=\left|\alpha^{-1}(\hat{S})\right| .
$$

Combined with line 8, we deduce that $\hat{N}=\alpha^{-1}(\hat{S})$.

By Lemma 5, for each $r \in\left(s_{j}\right)_{j \in N^{\prime}}$ there is a path $t \stackrel{1}{\rightarrow} t^{2} \stackrel{2}{\rightarrow} \cdots \stackrel{n}{\rightarrow} r \subseteq \Gamma\left(R ; p^{*}(R), \alpha\right)$ such that $t:=\alpha\left(i^{1}\right)$ is exhausted at $(x, \alpha)$. Note that if $D_{S}\left(R_{k}, p^{*}(R)\right) \cap \hat{S} \neq \emptyset$, then $D_{s}\left(R_{k}, p^{*}(\bar{R})\right) \subseteq \hat{S}$ and $F^{*}\left(R^{d}\right) P_{k} F^{*}(R)$. Therefore, for each $k \notin \hat{N}$, since $k \notin N^{\prime}, D_{S}\left(R_{k}, p^{*}(R)\right) \cap \hat{S}=\emptyset$. It follows that, since $r \in \hat{S}, i^{n} \in \hat{N}$. Then $\alpha\left(i^{n}\right) \in \hat{S}$, and it follows by the same argument that $i^{n-1} \in \hat{N}$, and so on. Conclude that $\left\{i^{1}, \ldots, i^{n}\right\} \subseteq \hat{N}$ and therefore that $t \in \hat{S}$. Since $t$ is exhausted at $(x, \alpha)$, it has endowment divisible value at $p^{*}(R)$ and $\left|\alpha^{-1}(t)\right|=c_{t}\left(p^{*}(R)\right)$. Moreover, $c_{t}(\bar{R})=c_{t}(R)-1$. Therefore, $\left|\alpha^{-1}(t)\right|>c_{t}(\bar{R})$. Thus, $c_{t}\left(p^{*}(\bar{R})\right)=\left|\alpha^{-1}(t)\right|-1$. Therefore, since $\hat{N}=\alpha^{-1}(\hat{S})$, line (9) 
then implies

$$
|\hat{N}|=\left|\alpha^{-1}(\hat{S})\right|>\sum_{\hat{s} \in \hat{S}} c_{\hat{S}}\left(p^{*}(\bar{R})\right),
$$

a contradiction.

With Lemma 2 shown, we may now use the fact that $F^{*}$ is weakly group-strategy-proof.

Proof of Property 1. By Property 2, we may confine attention to the case when $s \in D_{S}\left(R_{i}, \pi(0)\right)$. Suppose that for $d>0, \pi_{s}(d)<\pi_{s}(0)$. If $s \notin D_{S}\left(R_{i}^{d}, \pi(d)\right)$, then we apply Property 2 to conclude that $\pi_{s}(0)=\pi_{s}(d)<\pi_{s}(0)$. Therefore, $s \in D_{S}\left(R_{i}^{d}, \pi(d)\right)$. By definition of $R^{d}$, for each pair of bundles $(x, s)$ and $(y, t)$ with $t \neq s$, if $(x, s) I_{i}^{d}(y, t)$ then $(y, t) P_{i}(x, s)$. Therefore, for each $\alpha \in \mathcal{A}^{*}\left(R^{d}\right)$,

$$
\llbracket \pi(d), \alpha(i) \rrbracket R_{i}\left(\frac{w}{\pi_{s}(d)}, s\right) P_{i} F^{*}(R),
$$

contradicting strategy-proofness.

\section{Appendix B. Proof of Theorem 5}

Proposition 2. $F^{*}$ satisfies ${ }$-Con.

Proof. Briefly consider an alternate definition of equilibrium. Triple $(p, x, \alpha)$ is a constrained price equilibrium if it is an equilibrium and, for each site $s \in S$ with $\alpha^{-1}(s)=\emptyset, p_{s}=w e_{s}^{-1}$. Given an equilibrium, $(q, y, \beta)$, define for each $s \in S, p_{s}:=\max \left\{q_{s}, w e_{s}^{-1}\right\}$. It is easy to verify that $(p, y, \beta)$ is a constrained price equilibrium. It follows that $p^{* *}(R)$ defined from $p^{*}(R)$ by this mapping is the unique minimal constrained equilibrium price for economy $R$. Let $F^{* *}$ be the rule such that each $F^{* *}(R)$ is the set of equilibria supported by $p^{* *}(R)$. If there is $\alpha \in \mathcal{A}^{*}(R)$ and $s \in S$ with $\alpha^{-1}(s) \neq \emptyset$, then feasibility implies $p_{s}^{*}(R) \geq w e_{s}^{-1}$, which further implies $p_{s}^{* *}(R)=p_{s}^{*}(R)$. Thus, $F^{* *}=F^{*}$.

Let $f \in F^{*}$. Let $R^{n} \in \mathcal{R}^{N}$ be a sequence converging to $R \in \mathcal{R}^{N}$. Let $(x, \alpha)$ be an allocation such that for each $n \in \mathbb{N}, f\left(R^{n}\right)=(x, \alpha)$. Let $\hat{S}:=\alpha(N)$. For each $s \notin \hat{S}, p_{s}^{* *}(R)=w e_{s}^{-1}$. Clearly, for each $s \in \hat{S}, p_{s}^{* *}\left(R^{n}\right)$ is a constant sequence. Define $\bar{p}:=p^{* *}\left(R^{n}\right)$. By Lemma 3 , there is $\bar{n} \in \mathbb{N}$ such that for each $n>\bar{n}$, and each $i \in N, D_{S}\left(R_{i}, \bar{p}\right) \supseteq D_{S}\left(R_{i}^{n}, \bar{p}\right)$. This implies moreover that $\bar{p} \in \mathbb{P}(R)$ and therefore, $p^{* *}(R) \leq \bar{p}$.

Let $n \geq \bar{n}$. Construct preference relation $\hat{R}_{1}$ such that $D_{S}\left(\hat{R}_{1}, \bar{p}\right)=D_{S}\left(R_{1}, \bar{p}\right)$ by performing successive, positive site-translations. By Property $2, p^{* *}\left(\hat{R}_{1}, R_{-1}^{n}\right)=\bar{p}, p^{* *}\left(\hat{R}_{1}, \hat{R}_{2}, R_{N \backslash\{1,2\}}^{n}\right)=\bar{p}$ 
and so on. Conclude that $p^{* *}(\hat{R})=\bar{p}$. Next, for each $i$, construct $\tilde{R}_{i}$ from $\hat{R}_{i}$ by site translations such that $(x, s) \tilde{I}_{i} D\left(\tilde{R}_{i}, \bar{p}\right)$ if and only if $(x, s) I_{i} D\left(R_{1}, \bar{p}\right)$. That is, the optimizing indifference set of $\tilde{R}_{i}$ for prices $\bar{p}$ is identical to the optimizing indifference set of $R_{i}$ for prices $\bar{p}$. Since we have already set $D_{S}\left(\hat{R}_{i}, \bar{p}\right)=D_{S}\left(R_{i}, \bar{p}\right)$, this operation involves sites $s \notin D_{S}\left(\hat{R}_{i}, \bar{p}\right)$. Therefore, by Property 2 again conclude that $p^{* *}(\tilde{R})=\bar{p}$. By strategy-proofness, $f\left(R_{1}, \tilde{R}_{-1}\right) R_{1} f(\tilde{R})$. If $f\left(R_{1}, \tilde{R}_{-1}\right) P_{1} f(\tilde{R})$, then by construction, $f\left(R_{1}, \tilde{R}_{-1}\right) \tilde{P}_{1} f(\tilde{R})$, contradicting strategy-proofness. Therefore, $f\left(R_{1}, \tilde{R}_{-1}\right) I_{1} f(\tilde{R})$. It follows that, since the preferences of other agents remain constant, $f\left(R_{1}, \tilde{R}_{-1}\right)$ is an equilibrium for $\tilde{R}$ and $\bar{p}=p^{* *}(\tilde{R}) \leq p^{* *}\left(R_{1}, \tilde{R}_{-1}\right)$. We conclude then that $p^{* *}\left(R_{1}, \tilde{R}_{-1}\right)=\bar{p}$. Proceed inductively to conclude that $p^{* *}(R)=p^{* *}(\tilde{R})=\bar{p}$.

B.1. The domain of unique assignment cardinality. For each $k \in \mathbb{Z}_{+}$, define

$$
\mathcal{D}^{k}:=\left\{R \in \mathcal{R}^{N}: \sum c_{s}\left(p^{*}(R)\right) \leq|N|+k\right\} .
$$

If $R \in \mathcal{D}^{0}$, then $\sum_{s \in S} c_{s}\left(p^{*}(R)\right)=|N|$. It follows that for each $\alpha \in \mathcal{A}^{*}(R)$ and each $s \in S$, $\left|\alpha^{-1}(s)\right|=c_{s}\left(p^{*}(R)\right)$. Therefore, we refer to $\mathcal{D}^{0}$ as the domain of unique assignment cardinality or the domain of unique size for short.

In service of proving the main theorem, we first prove

Proposition 3. Let $\varphi$ be a single-valued rule. Assume $\varphi$ is strategy-proof, welfare anonymous, constant sequence continuous, and strongly undominated in these properties. Then for each $R \in$ $\mathcal{D}^{0}, \varphi(R) \in F^{*}(R)$.

Without loss of generality, we assume in all that follows that $w=1$ and $e \equiv \mathbf{1}$.

A single-valued rule $\varphi$ is individually invariant to unilateral monotonic transformation, or unilaterally invariant for short, if for each $R \in \mathcal{R}^{N}$, and each $i \in N$, if $\left(x_{i}, s\right)=\varphi_{i}(R)$, then for each $R_{i}^{\prime} \in \mathcal{T}\left(R_{i},\left(x_{i}, s\right)\right),\left(x_{i}, s\right)=\varphi_{i}\left(R_{i}^{s, d}, R_{-i}\right)$. There is a well-known result in the literature, called the "Invariance Lemma" by Thomson (2014), that implies each strategy-proof rule is unilaterally invariant. Unilateral invariance, while being implied by strategy-proofness, is in fact closely related to strategy-proofness. See Klaus and Bochet (2013) for a thorough study.

Let $R \in \mathcal{D}^{0}$ and $p:=p^{*}(R)$. Let $\bar{c} \in \mathbb{N}^{S}$ be the list of numbers such that $\llbracket p, \alpha \rrbracket \in F^{*}(R)$ implies, for each $s \in S$, that $\left|\alpha^{-1}(s)\right|=\bar{c}_{s}$. Let $R_{0}^{p}$ be a preference relation such that $B$, the budget set given by prices $p$, is an indifference set of $R_{0}^{p}$. That is, given prices $p$, an agent with preferences $R_{0}^{p}$ is indifferent as to which site's commodity he wants to consume. Since $\mathcal{R}$ is sufficiently rich, $R_{0}^{p} \in \mathcal{R}$. Let $R^{p} \in \mathcal{R}^{N}$ be the profile such that for each $i \in N, R_{i}^{p}=R_{0}^{p}$. 
By repeated applications of Property 2, conclude that $p^{*}\left(R^{p}\right)=p$. Since $R \in \mathcal{D}^{0},|N|=$ $\sum_{s \in S} c_{s}\left(p^{*}(R)\right)=\sum_{s \in S} c_{s}\left(p^{*}\left(R^{p}\right)\right)$, and thus $R^{p} \in \mathcal{D}^{0}$.

Welfare anonymity implies that all agents consume on a common indifference set. Together with strong-undomination we deduce that there is $\beta \in S^{N}$ such that $\varphi\left(R^{p}\right)=\llbracket p, \beta \rrbracket$, and therefore, $\varphi\left(R^{p}\right) \in F^{*}\left(R^{p}\right)$. Note that, typically, $F^{*}\left(R^{p}\right) \supsetneq F^{*}(R)$; this fact accounts for much of the work in proving the following

Lemma. Let $i \in N$ and $\hat{R}:=\left(R_{-i}, R_{i}^{p}\right)$. For each $j \in N, \varphi_{j}(\hat{R}) \in D\left(\hat{R}_{j}, p\right)$.

Proof. Let $(x, \alpha) \in F^{*}(R)$ and let $i^{*} \in N$ be such that $s^{*}:=\alpha\left(i^{*}\right)$ is exhausted at $(x, \alpha)$. For each $\varepsilon>0$ and each $j \neq i^{*}$, let $R_{j}^{\varepsilon} \in \mathcal{R}$ satisfy $H^{\Delta}\left(R_{j}, R_{j}^{\varepsilon}\right)<\varepsilon$ and $R_{j}^{\varepsilon} \in \mathcal{T}\left(R_{j},\left(x_{j}, \alpha(j)\right)\right)$. We first show by induction that the lemma is true for profile $\left(\left(R_{j}^{\varepsilon}\right)_{j \neq i^{*}}, R_{i^{*}}^{p}\right)$.

Inductive Base: We showed above that there is $\beta \in S^{N}$ with $\varphi\left(R^{p}\right)=\llbracket p, \beta \rrbracket$. Since $R^{p} \in \mathcal{D}^{0}$, and $p^{*}\left(R^{p}\right)=p^{*}(R)$, for each $s \in S,\left|\beta^{-1}(s)\right|=\left|\alpha^{-1}(s)\right|$. It follows that for each $i \in N \backslash i^{*}$, there is $j \in \beta^{-1}(\alpha(i))$, which further implies that $\varphi_{j}\left(R^{p}\right)=\left(x_{i}, \alpha(i)\right)$. Let $\bar{R}_{j}:=R_{i}^{\varepsilon}$ and $(z, \gamma):=\varphi\left(\bar{R}_{j}, R_{-j}^{p}\right)$. By unilateral invariance, $\left(z_{j}, \gamma(j)\right)=\left(x_{i}, \alpha(i)\right)$. By welfare anonymity, $\varphi_{i}\left(R_{i}^{\varepsilon}, R_{-i}^{p}\right) I_{i}^{\varepsilon} \varphi_{j}\left(\bar{R}_{j}, R_{-j}^{p}\right)$ and therefore $\varphi_{i}\left(R_{i}^{\varepsilon}, R_{-i}^{p}\right) R_{i}^{\varepsilon} B$. By strategy-proofness, $B I_{i}^{p} \varphi_{i}\left(R^{p}\right) R_{i}^{p} \varphi_{i}\left(R_{i}^{\varepsilon}, R_{-i}^{p}\right)$. Since $R_{i}^{\varepsilon} \in$ $\mathcal{T}\left(R_{i},\left(x_{i}, \alpha(i)\right)\right) \subseteq \mathcal{T}\left(R^{p},\left(x_{i}, \alpha(i)\right)\right)$, conclude that $\varphi_{i}\left(R_{i}^{\varepsilon}, R_{-i}^{p}\right)=\left(x_{i}, \alpha(i)\right)$.

For each $k \in N \backslash i, F^{*}\left(R_{i}^{\varepsilon}, R_{-i}^{p}\right) I_{k}^{p} B$, therefore by strong-undomination, there exists $k^{\prime} \in N \backslash i$ such that $\varphi_{k^{\prime}}\left(R_{i}^{\varepsilon}, R_{-i}^{p}\right) R_{k^{\prime}}^{p} B$. By welfare anonymity, for each $k \in N \backslash i,\left(z_{k}, \gamma(k)\right) I_{k}^{p}\left(z_{k^{\prime}}, \gamma\left(k^{\prime}\right)\right)$. In sum, for each $k \in N, \varphi_{k}\left(R_{i}^{\varepsilon}, R_{-i}^{p}\right) R_{k}^{p} B$. Since preferences are increasing, $z_{k} \geq \frac{1}{p_{\gamma(k)}}$, where we recall that, given our normalization, $\frac{1}{p_{\gamma(k)}}=\frac{w}{p_{\gamma(k)}}$. It follows by feasibility that $\left|\gamma^{-1}(\gamma(k))\right| \leq c_{\gamma(k)}(p)=$ $\left|\alpha^{-1}(\gamma(k))\right|$. Since $k$ is arbitrary, for each $s \in S,\left|\gamma^{-1}(s)\right| \leq\left|\alpha^{-1}(s)\right|$. Now since, for each $s \in S$, $\left|\alpha^{-1}(s)\right|=\bar{c}_{s}$, and since $\sum \bar{c}_{s}=|N|$, it follows that $\left|\gamma^{-1}(s)\right|=\left|\alpha^{-1}(s)\right|$. Since $i \neq i^{*}$, this further implies there is $k^{*} \in N \backslash i$ satisfying $\gamma\left(k^{*}\right)=s^{*}$ and $z_{k^{*}} \geq x_{i^{*}}{ }^{12}$ Since for each $k \in \gamma^{-1}\left(s^{*}\right), z_{k} \geq x_{i^{*}}$, by feasibility, $z_{k^{*}}=x_{i^{*}}$ (recall that $s^{*}=\alpha\left(i^{*}\right)$ is exhausted at $\left.(x, \alpha)\right)$. Since $k^{*}$ 's preferences are $R_{0}^{p}$, welfare anonymity implies that for each $k \in N \backslash i, \varphi_{k}\left(R_{i}^{\varepsilon}, R_{-i}^{p}\right) \in B$.

Induction Step: Fix $n \in \mathbb{N}$. The induction hypothesis is as follows: Let $\hat{R} \in \mathcal{R}^{N}$ and $N^{\prime}:=$ $\left\{i \in N: \hat{R}_{i} \neq R_{0}^{p}\right\}$. Assume that $\left|N^{\prime}\right| \leq n, i^{*} \notin N^{\prime}$ and, for each $i \in N^{\prime}, \hat{R}_{i}=R_{i}^{\varepsilon}$. Then, for each $j \in N, \varphi_{j}(\hat{R}) \in D\left(\hat{R}_{j}, p\right) \subseteq B$.

$\overline{{ }^{12} \text { If } \mid \gamma^{-1}\left(\alpha\left(i^{*}\right)\right)} \mid=1$, then since $\left|\gamma^{-1}\left(\alpha\left(i^{*}\right)\right)\right|=\left|\alpha^{-1}\left(\alpha\left(i^{*}\right)\right)\right|$, we have $\alpha^{-1}\left(\alpha\left(i^{*}\right)\right)=\left\{i^{*}\right\}$. Since $i \neq i^{*}, \alpha(i) \neq \alpha\left(i^{*}\right)$, and therefore $\gamma(j) \neq \alpha\left(i^{*}\right)$. 
Let $\hat{R}$ satisfy the induction hypothesis and let $(y, \beta):=\varphi(\hat{R})$. For compact notation, let $R_{N^{\prime}}^{\varepsilon}$ denote the partial profile $\left(R_{i}^{\varepsilon}\right)_{i \in N^{\prime}}$.

Claim 4. For each $i \in N \backslash N^{\prime}$, there is $j \in N \backslash N^{\prime}$ such that $\beta(j)=\alpha(i)$.

Proof. Let $\alpha(i)=s$. The claim is thus

$$
i \in N \backslash N^{\prime} \Longrightarrow\left(\exists j \in N \backslash N^{\prime} \text { s.t. } \beta(j)=s\right) .
$$

Since $\varphi(\hat{R})$ assigns only bundles in $B$, for each $s^{\prime} \in S,\left|\beta^{-1}\left(s^{\prime}\right)\right| \leq \bar{c}_{s^{\prime}}$. Since $\sum \bar{c}_{s^{\prime}}=|N|$, feasibility implies that in fact $\left|\beta^{-1}\left(s^{\prime}\right)\right|=\bar{c}_{s^{\prime}}$. For each $k \in N^{\prime}$, since $\left\{\left(x_{k}, \alpha(k)\right)\right\}=D\left(R_{k}^{\varepsilon}, p\right)$, the induction hypothesis that $\left(y_{k}, \beta(k)\right) \in D\left(R_{k}^{\varepsilon}, p\right)$ yields $\left(y_{k}, \beta(k)\right)=\left(x_{k}, \alpha(k)\right)$. Thus, $\left(\beta^{-1}(s) \cap N^{\prime}\right) \subseteq \alpha^{-1}(s)$.

The contrapositive hypothesis is $\beta^{-1}(s) \subset N^{\prime}$. Then $\beta^{-1}(s) \subseteq \alpha^{-1}(s)$, and we deduce

$$
\bar{c}_{s}=\left|\beta^{-1}(s)\right|=\left|\alpha^{-1}(s) \cap \beta^{-1}(s)\right| \leq\left|\alpha^{-1}(s) \cap N^{\prime}\right| .
$$

Since $\bar{c}_{s}=\left|\alpha^{-1}(s)\right|$ by definition, this further implies $\alpha^{-1}(s) \subseteq N^{\prime}$ and $i \in N^{\prime}$.

Let $i \in N \backslash\left(N^{\prime} \cup i^{*}\right)$. By Claim 4, there is $j \in N \backslash N^{\prime}$ such that $\beta(j)=\alpha(i)$. Let $\bar{R}_{j}:=R_{i}^{\varepsilon}$, and denote $\bar{R}:=\left(\bar{R}_{j}, \hat{R}_{-j}\right)$. Let $(z, \gamma):=\varphi(\bar{R})$. Unilateral invariance implies that $\left(z_{j}, \gamma(j)\right)=\left(x_{i}, \alpha(i)\right)$. By welfare anonymity, $\varphi_{i}\left(R_{i}^{\varepsilon}, \hat{R}_{-i}\right) I_{i}^{\varepsilon} \varphi_{j}\left(\bar{R}_{j}, \hat{R}_{-j}\right)$ and therefore $\varphi_{i}\left(R_{i}^{\varepsilon}, \hat{R}_{-i}\right) R_{i}^{\varepsilon} B$. By strategyproofness, $B I_{i}^{p} \varphi_{i}(\hat{R}) R_{i}^{p} \varphi_{i}\left(R_{i}^{\varepsilon}, \hat{R}_{-i}\right)$. Since $R_{i}^{\varepsilon} \in \mathcal{T}\left(R_{i},\left(x_{i}, \alpha(i)\right)\right) \subseteq \mathcal{T}\left(R^{p},\left(x_{i}, \alpha(i)\right)\right)$, conclude that $\varphi_{i}\left(R_{i}^{\varepsilon}, \hat{R}_{-i}\right)=\left(x_{i}, \alpha(i)\right)$.

Claim 5. For each $k \in N$,

$$
\left(z_{k}, \gamma(k)\right) \hat{R}_{k} D\left(\hat{R}_{k}, p\right)
$$

Proof. We have shown the claim for $i$. The proof is by contradiction. Assume there are $k \in N$ and $\left(y_{k}, r\right) \in D\left(\hat{R}_{k}, p\right)$ such that

$$
\left(y_{k}, r\right) \hat{P}_{k}\left(z_{k}, \gamma(k)\right) \text {. }
$$

Assume first that $k \in N \backslash\left(N^{\prime} \cup i\right)$. Thus, $k^{\prime}$ s preferences are $R_{0}^{p}$. Since for each $k^{\prime} \in N$ and each $f \in F^{*}, f\left(R_{i}^{\varepsilon}, \hat{R}_{-i}\right) \hat{I}_{k^{\prime}} D\left(\hat{R}_{k^{\prime}}, p\right)$, strong undomination implies there is $k^{\prime \prime} \in N \backslash i$ for whom

$$
\left(z_{k^{\prime \prime}}, \gamma\left(k^{\prime \prime}\right)\right) \hat{P}_{k^{\prime \prime}} D\left(\hat{R}_{k^{\prime \prime}}, p\right)
$$

which further implies, since preferences are increasing and $w=1$, that $z_{k^{\prime \prime}}>p_{\gamma\left(k^{\prime \prime}\right)}^{-1}$. Welfare anonymity implies that the preferences of $k$ and $k^{\prime \prime}$ differ and therefore that $k^{\prime \prime} \in N^{\prime}$. Profile $\bar{R}:=\left(R_{k^{\prime \prime}}^{p}, R_{i}^{\varepsilon}, \hat{R}_{-i-k^{\prime \prime}}\right)$ satisfies the induction hypothesis and therefore, for each $i \in N, \varphi_{i}(\bar{R}) \in$ 
$D\left(\bar{R}_{i}, p\right) \subset B$. Then if $\bar{R}$ is the true profile, $k^{\prime \prime}$ will manipulate by reporting $R_{k^{\prime \prime}}^{\varepsilon}$, contradicting strategy-proofness. Conclude that $k \notin N \backslash\left(N^{\prime} \cup i\right)$.

Assume now that $k \in N^{\prime} ; k^{\prime}$ s preferences are $R_{k}^{\varepsilon}$. Profile $\bar{R}:=\left(R_{k}^{p}, R_{i}^{\varepsilon}, \hat{R}_{-i-k}\right)$ satisfies the induction hypothesis and therefore $\varphi(\bar{R}) \in B^{N}$. We also apply Claim 4: there is $k^{\prime} \in N$ with preferences $R_{0}^{p}$ such that $\varphi_{k^{\prime}}\left(R_{k}^{p}, \bar{R}_{-k}\right)=\left(x_{k}, \alpha(k)\right)$. Let $\tilde{R}_{k^{\prime}}:=R_{k}^{\varepsilon}$ and denote $\tilde{R}:=\left(\tilde{R}_{k^{\prime}}, \bar{R}_{-k^{\prime}}\right)$. By unilateral invariance, $\varphi_{k^{\prime}}(\tilde{R})=\left(x_{k}, \alpha(k)\right)$. However, since $\left(x_{k}, \alpha(k)\right) \in D\left(\hat{R}_{k}, p\right)$,

$$
\left(y_{k}, r\right) I_{k}\left(x_{k}, \alpha(k)\right) P_{k}\left(z_{k}, \gamma(k)\right) \text {, }
$$

contradicting welfare anonymity. In sum, we have deduced that $k \notin N$.

Let $k \in N^{\prime}$. If $\varphi_{k}\left(R_{i}^{\varepsilon}, \hat{R}_{-i}\right)$ is above $B$, then by the induction hypothesis, at profile $\left(R_{i}^{\varepsilon}, R_{k}^{p}, \hat{R}_{-i-k}\right)$, agent $k$ successfully manipulates the rule by reporting $R_{k}^{\varepsilon}$. Therefore, for each $k \in N^{\prime}, \varphi_{k}\left(R_{i}^{\varepsilon}, \hat{R}_{-i}\right) \in$ $D\left(\hat{R}_{i}, p\right) \subseteq B$. Strong undomination together with welfare anonymity imply that for each $k \in$ $N \backslash N^{\prime}, \varphi_{k}\left(R_{i}^{\varepsilon}, \hat{R}_{-i}\right) \hat{R}_{k} B$.

Since preferences are increasing, for each $k \in N, z_{k} \geq p_{\gamma(k)}^{-1}$. It follows by feasibility that $\left|\gamma^{-1}(\gamma(k))\right| \leq\left|\alpha^{-1}(\gamma(k))\right|$, and since $k$ was arbitrary, for each $s \in S,\left|\gamma^{-1}(s)\right| \leq\left|\alpha^{-1}(s)\right|$. Now since, for each $s \in S,\left|\alpha^{-1}(s)\right|=\bar{c}_{s}$, and since $\sum \bar{c}_{s}=|N|$, it follows that $\left|\gamma^{-1}(s)\right|=\left|\alpha^{-1}(s)\right|$.

Suppose that $\gamma^{-1}\left(s^{*}\right) \subseteq N^{\prime} \cup i$. Let $k \in \gamma^{-1}\left(s^{*}\right)$, and since $k \in N^{\prime} \cup i,\left(R_{i}^{\varepsilon}, \hat{R}_{-i}\right)_{k}=R_{k}^{\varepsilon}$. We established that $\varphi_{k}\left(R_{i}^{\varepsilon}, \hat{R}_{-i}\right) \in D\left(R_{k}^{\varepsilon}, p\right)=\left\{\left(x_{k}, \alpha(k)\right)\right\}$. Therefore, $\varphi_{k}\left(R_{i}^{\varepsilon}, \hat{R}_{-i}\right)=\left(x_{k}, \alpha(k)\right)$. Thus, $s^{*}=\gamma(k)=\alpha(k)$. As above, we deduce

$$
\bar{c}_{s^{*}}=\left|\gamma^{-1}\left(s^{*}\right)\right|=\left|\gamma^{-1}\left(s^{*}\right) \cap\left(N^{\prime} \cup i\right)\right| \leq\left|\alpha^{-1}(s) \cap\left(N^{\prime} \cup i\right)\right| .
$$

Since $\alpha^{-1}\left(s^{*}\right)=\bar{c}_{s^{*}}$, conclude that $\alpha^{-1}\left(s^{*}\right) \subseteq\left(N^{\prime} \cup i\right)$. But this implies $i^{*} \in N^{\prime} \cup i$, a contradiction.

Therefore, there is an agent $k \in N \backslash N^{\prime}$ such that $\gamma(k)=\alpha\left(i^{*}\right)$. By feasibility, $\varphi_{k}\left(R_{i}^{\varepsilon}, \hat{R}_{-i}\right)=$ $\llbracket p, \gamma(k) \rrbracket$ and it follows from welfare anonymity that, for each $k \in N \backslash N^{\prime}, \varphi_{k}\left(R_{i}^{\varepsilon}, \hat{R}_{-i}\right) \in B$.

Now let $i \in N$ be arbitrary. If $\alpha(i)$ is not exhausted at $\llbracket p, \alpha \rrbracket$, then there exists a path $r \rightsquigarrow \alpha(i) \subset$ $\Gamma(R ; p, \alpha)$ such that $r$ is exhausted at $\llbracket p, \alpha \rrbracket$. Let $\alpha^{\prime}$ be the path shift of $\alpha$ via $r \rightsquigarrow \alpha(i)$ and define $\alpha^{*}$ so that, for each $j \in N$,

$$
\alpha^{*}(j):= \begin{cases}r & j=i \\ \alpha^{\prime}(j) & \text { otherwise }\end{cases}
$$


Note that $p^{*}\left(R_{i}^{p}, R_{-i}\right)=p^{*}(R)$ and $\alpha^{*} \in \mathcal{A}^{*}\left(R_{i}^{p}, R_{-i}\right)$. Therefore, our argument holds for profile $\left(R_{i}^{p}, R_{-i}\right)$ by setting $i^{*}=i$ and using site assignment $\alpha^{*}$. Conclude that for each $j \neq i, \varphi_{j}\left(R_{i}^{p}, R_{-i}^{\varepsilon}\right) \in$ $D\left(R_{j}, p\right)$ and $\varphi_{i}\left(R_{i}^{p}, R_{-i}^{\varepsilon}\right) \in D\left(R_{i}^{p}, p\right)$.

We have shown that for each $i \in N$, each site assignment $\alpha \in S^{N}$ satisfying either $\alpha \in \mathcal{A}^{*}(R)$ or $\alpha$ is constructed as $\alpha^{*}$, each $\varepsilon>0$, and each $j \neq i, \varphi_{j}^{i, \varepsilon}:=\varphi_{j}\left(\left(R_{j}^{\varepsilon}\right)_{j \neq i}, R_{i}^{p}\right) \in D\left(R_{j}^{\varepsilon}, p\right)$. Moreover, $\varphi_{i}^{i, \varepsilon}:=\varphi_{i}\left(\left(R_{j}^{\varepsilon}\right)_{j \neq i}, R_{i}^{p}\right) \in D\left(R_{i}^{p}, p\right)$. Therefore, there exists $\beta \in S^{N}$ such that $\varphi^{i, \varepsilon}=\llbracket p, \beta \rrbracket$. By construction, for each $j \neq i, \varphi_{j}^{i, \varepsilon}=\llbracket p, \alpha(j) \rrbracket$. Recall that $\sum_{s \in S} \bar{c}_{s}=\sum_{s \in S} c_{s}(p)=|N|$, which leaves $\varphi_{i}^{i, \varepsilon}=\llbracket p, \alpha(i) \rrbracket$. Since $\varepsilon>0$ was arbitrary, constant sequence continuity implies that $\varphi_{i}\left(R_{-i}, R_{i}^{p}\right) \in D\left(R_{i}^{p}, p\right)$ and, for each $j \neq i, \varphi_{j}\left(R_{-i}, R_{i}^{p}\right) \in D\left(R_{j}, p\right)$.

It remains only to show that at $\varphi(R)$, all agents are maximizing their $R$ preferences on $B$. If there is an agent $k$ with

$$
\varphi_{k}(R) P_{k} D\left(R_{k}, p\right)
$$

then since preferences are increasing, $\varphi_{k}(R)$ is above $B$. When $\left(R_{k}^{p}, R_{-k}\right)$ is the true profile, $k$ manipulates by reporting $R_{k}$, a contradiction. Thus we have that for each agent $i$,

$$
D\left(R_{i}, p\right) R_{i} \varphi_{i}(R) .
$$

But then by strong undomination we have for each agent $i$ that $\varphi_{i}(R) R_{i} D\left(R_{i}, p\right)$, and the proof is complete.

B.2. Proof of Theorem 5. A graph $\Gamma$ is simple if for each $(r, s) \in S \times S$, there is at most one $i \in N$ such that $(r, s, i) \in \Gamma$. Note that path shifting preserves simplicity. A tree is an acyclic, connected graph $\Gamma$ such that, for each $s \in S$, there is at most one $r \in S$ with $r \rightarrow s .{ }^{13}$ Thus, each tree has a unique vertex, called the root, with no incoming edges. A graph is a forest if it is the disjoint union of trees. A graph $\Gamma\left(R ; p^{*}(R), \alpha\right)$, generated by an equilibrium assignment $\llbracket p^{*}(R), \alpha \rrbracket$, is a minimal simple forest if it is a simple forest with the following property: for each tree $T \subseteq \Gamma\left(R ; p^{*}(R), \alpha\right)$, site $r \in T$ is exhausted at $\llbracket p^{*}(R), \alpha \rrbracket$ if and only if it is the root. For each $k \in \mathbb{Z}_{+}$, let $\mathcal{D}^{k-1 / 2} \subseteq \mathcal{D}^{k}$ be the set of all economies $R \in \mathcal{D}^{k}$ with $\alpha \in \mathcal{A}(R)$ such that $\Gamma\left(R ; p^{*}(R), \alpha\right)$ is a minimal simple forest. This forces us to live with the awkward notation $\mathcal{D}^{-1 / 2}$ for the elements of $\mathcal{D}^{0}$ with the

\footnotetext{
${ }^{13}$ This is a departure from the standard terminology. The word tree is usually reserved for undirected graphs, whereas the structure we describe is an arborescence. Since we are concerned only with directed graphs, we use the simpler term.
} 
simple-forest property. This cost is recouped in the proof of Theorem 5 below, which is just an exercise in cumbersome notation once Lemmas 10, 11, and 12 are shown.

Lemma 10. Assume $k>0$ and let $R \in \mathcal{D}^{k-1 / 2}$ and $p:=p^{*}(R)$. For each $i \in N$, there is a site $t \in S$ and a sequence $\left\{R_{i}^{n}\right\} \subseteq \mathcal{T}\left(R_{i}^{p}, \llbracket p, t \rrbracket\right)$ such that $R_{i}^{n} \rightarrow R^{p}$ and, for each $n \in \mathbb{N},\left(R_{i}^{n}, R_{-i}\right) \in \mathcal{D}^{k-1}$.

Proof. Let $i \in N, \alpha \in \mathcal{A}(R)$, and $s:=\alpha(i)$. Assume $\Gamma(R ; p, \alpha)$ is a minimal simple forest. Let $T \subseteq \Gamma(R ; p, \alpha)$ be the tree to which $s$ belongs and $r^{0}$ its root. There is $t \in S$, such that $\left|\alpha^{-1}(t)\right| \leq$ $c_{t}\left(p^{*}(R)\right)-1$ (otherwise $R \in \mathcal{D}^{0}$ ).

There is a unique path $r^{0} \leadsto s:=\left[r^{0} \rightarrow r^{1} \rightarrow \cdots \rightarrow\left(r^{m}:=s\right)\right]$ in $\Gamma(R ; \alpha)$ from $r^{0}$ to $s$. Assume first that $t \in\left(r^{l}\right)_{l=0}^{m}$, so there is $m^{\prime} \in\{0, \ldots, m\}$ such that $t=r^{m^{\prime}}$. Let $r^{0} \rightsquigarrow r^{m^{\prime}} \subseteq r^{0} \rightsquigarrow s$ be the subpath from $r^{0}$ to $r^{m^{\prime}}$. Let $\alpha^{\prime}$ be the path shift of $\alpha$ via $r^{0} \leadsto r^{m^{\prime}}$. Since $\left|\alpha^{-1}\left(r^{m^{\prime}}\right)\right| \leq c_{r^{m^{\prime}}}(p)-1$, $\alpha^{\prime}$ is feasible. Therefore, by Lemma $6, \alpha^{\prime} \in \mathcal{A}(R)$.

There is a tree $T^{\prime} \subseteq \Gamma\left(R ; p, \alpha^{\prime}\right)$ having the same vertex and undirected edge set as $T$, but with $r^{m^{\prime}}$ as the root. The unique path from $r^{m^{\prime}}$ to $s$ in $T^{\prime}$ is clearly the path $r^{m^{\prime}} \rightarrow r^{m^{\prime}+1} \rightarrow \cdots \rightarrow r^{m}$.

If there is $t^{\prime} \in\left(r^{l}\right)_{l=m^{\prime}}^{m}$ such that $\left|\alpha^{-1}\left(t^{\prime}\right)\right| \leq c_{t^{\prime}}(p)-1$, then we repeat the process in the previous paragraph to construct a site-assignment $\alpha^{\prime \prime} \in \mathcal{A}(R)$ and find that $s$ now belongs to tree $T^{\prime \prime}$ with $t^{\prime}$ as root. In sum, we may assume without loss of generality that we have chosen $\alpha \in \mathcal{A}(R)$ such that the site $t$ is not in $r^{0} \leadsto s$.

Fix $\varepsilon>0$ and let $R_{i}^{\varepsilon} \in \mathcal{T}\left(R_{i}^{p}, \llbracket p, t \rrbracket\right)$ satisfy $H^{\Delta}\left(R_{i}^{\varepsilon}, R_{i}^{p}\right)<\varepsilon$ (recall that since $\mathcal{R}$ is a rich domain, both $R_{i}^{p} \in \mathcal{R}$ and $\left.R_{i}^{\varepsilon} \in \mathcal{R}\right)$. Let $\beta \in S^{N}$ be given, for each $j \in N$, by $\beta(j)=t$ if $j=i$, and $\beta(j)=\alpha(j)$ otherwise. Since $\left|\alpha^{-1}(t)\right| \leq c_{t}(p)-1, \llbracket p, \beta \rrbracket$ is an equilibrium for $R^{\prime}:=\left(R_{i}^{\varepsilon}, R_{-i}\right)$, so $p \in \mathbb{P}\left(R^{\prime}\right)$.

Since $\alpha(i)=s$, by construction, $i$ labels no arcs in the path $r^{0} \rightsquigarrow s$. Therefore, it is still the case that $r^{0} \rightsquigarrow s \subseteq \Gamma\left(R^{\prime} ; p, \beta\right)$. Let $\gamma$ be the path shift of $\beta$ via $r^{0} \leadsto s$. Since $i$ has left $s, \llbracket p, \gamma \rrbracket$ is feasible. Then, by Lemma $6, \llbracket p, \gamma \rrbracket$ is an equilibrium for $R^{\prime}$. In $\Gamma\left(R^{\prime} ; p, \gamma\right)$, the path $r^{0} \rightsquigarrow s$ has been reversed: $s \rightsquigarrow r^{0} \subset \Gamma(R ; p, \gamma)$.

We construct $p^{\prime} \in \mathbb{R}^{S}$ such that for each $r^{l} \in s \leadsto r^{0}$, there is $\eta^{l}>0$ with $p_{s}^{\prime}=\left(1-\eta^{l}\right) p_{s}$, and for each $t^{\prime} \notin s \rightsquigarrow r^{0}, p_{t^{\prime}}^{\prime}=p_{t^{\prime}}^{*}(R)$. Our construction is inductive, so for each $l \in\{0, \ldots, m\}$ and each $t^{\prime} \in S$, let

$$
p_{t^{\prime}}^{l}:= \begin{cases}\left(1-\eta^{n}\right) p_{t^{\prime}} & \exists n \geq l, t^{\prime}=r^{n} \\ p_{t^{\prime}} & \text { otherwise. }\end{cases}
$$

Note that if $r^{0}=s$, then the path $r^{0} \leadsto s \subset \Gamma(R ; p, \alpha)$ was trivial, and now at $\llbracket p, \gamma \rrbracket, s$ is not exhausted, not blocked by indifference by an exhausted site, and satisfies $\left|\gamma^{-1}(s)\right| \leq c_{s}(p)-1$. Thus 
we may lower the price of $s$ and maintain feasibility. Otherwise, since $\Gamma(R ; p, \alpha)$ is a minimal simple tree, $s$ was not exhausted at $\llbracket p, \alpha \rrbracket$ and remains unexhausted at $\llbracket p, \gamma \rrbracket$. Thus, in the following arguments, setting each $\eta^{n}$ sufficiently small retains feasibility.

Since there are no arcs in $\Gamma\left(R^{\prime} ; p^{*}(R), \gamma\right)$ ending at $s$ ( $s$ is now the root of a tree), we may set $\eta^{m}$ sufficiently small that if $s \in D\left(R_{j}^{\prime} ; p^{m}\right)$ then $\gamma(j)=s$. Since the price of $s$ has decreased, for each $j \in \gamma^{-1}(s), r^{m-1} \notin D\left(R_{j}^{\prime} ; p^{m}\right)$. Furthermore, since $\left(s=r^{m}\right) \rightarrow r^{m-1} \in \Gamma\left(R^{\prime} ; p, \gamma\right)$, and $\Gamma\left(R^{\prime} ; p, \gamma\right)$ is a simple graph, for each $j^{\prime} \notin \gamma^{-1}(s) \cup \gamma^{-1}\left(r^{m-1}\right), r^{m-1} \notin D\left(R_{j^{\prime}}^{\prime} ; p^{*}(R)\right)$. Then by construction, it remains the case that $r^{m-1} \notin D\left(R_{j^{\prime}}^{\prime} ; p^{m}\right)$. Thus we may set $\eta^{m-1}$ sufficiently small that if $r^{m-1} \in$ $D_{S}\left(R_{k}^{\prime} ; p^{m-1}\right)$ then $r^{m-1}=\gamma(k)$. Continue inductively to define each $\eta^{l}$, for $l \in\{0,1, \ldots, m\}$ and therefore define $p^{\prime}$.

We now show that $p^{\prime} \in \mathbb{P}\left(R^{\prime}\right)$. Let $j \in \gamma^{-1}\left(\left\{r^{0}, r^{1}, \ldots, s\right\}\right)$. By construction, $D_{S}\left(R_{j}^{\prime} ; p^{\prime}\right)=$ $\{\gamma(j)\}$. Now let $j \in N \backslash \gamma^{-1}\left(\left\{r^{0}, r^{1}, \ldots, s\right\}\right)$. Then since there are no arcs $r \stackrel{j}{\rightarrow} r^{\prime} \in \Gamma\left(R^{\prime} ; p, \beta\right)$ with $r^{\prime} \in\left\{r^{0}, r^{1}, \ldots, s\right\}, D_{S}\left(R_{j} ; p\right) \cap\left\{r^{0}, r^{1}, \ldots, s\right\}=\emptyset$. By construction, since each $\eta^{l}$ is small, $D_{S}\left(R_{j} ; p^{\prime}\right) \cap\left\{r^{0}, r^{1}, \ldots, s\right\}=\emptyset$. Since the prices of the sites $S \backslash\left\{r^{0}, r^{1}, \ldots, s\right\}$ remain unchanged under $p, D\left(R_{j}^{\prime} ; p^{\prime}\right)=D\left(R_{j}^{\prime} ; p\right)$. We conclude that $\llbracket p^{\prime}, \gamma \rrbracket$ is an equilibrium for $R^{\prime}$ and therefore that $p^{\prime} \in \mathbb{P}\left(R^{\prime}\right)$.

Since $r^{0}$ is exhausted at $\llbracket p, \alpha \rrbracket,\left|\alpha^{-1}\left(r^{0}\right)\right|=\tilde{c}_{r^{0}}(p)$, and therefore $c_{r^{0}}(p)=\tilde{c}_{r^{0}}(p)$. Since, by construction, $\left|\gamma^{-1}\left(r^{0}\right)\right|=\left|\alpha^{-1}\left(r^{0}\right)\right|-1$, we have $\left|\gamma^{-1}\left(r^{0}\right)\right| \leq \tilde{c}_{r^{0}}(p)-1$. Recall that, given our normalizations $(w=1$ and $e \equiv \mathbf{1}), \tilde{c}_{r^{0}}(p)=\left\lfloor p_{r^{0}}\right\rfloor$, and a necessary condition for $r^{0}$ to be exhausted is that $p_{r^{0}} \in \mathbb{Z}$. Therefore, since $p_{r^{0}}^{\prime}<p_{r^{0}},\left\lfloor p_{r^{0}}^{\prime}\right\rfloor \leq\left\lfloor p_{r^{0}}\right\rfloor-1=p_{r^{0}}-1$, implying $\tilde{c}_{r^{0}}\left(p^{\prime}\right) \leq \tilde{c}_{r^{0}}(p)-1=$ $c_{r^{0}}(p)-1 \leq c_{r^{0}}$. Therefore, $c_{r^{0}}\left(p^{\prime}\right)=\tilde{c}_{r^{0}}\left(p^{\prime}\right)$ and $c_{r^{0}}\left(p^{\prime}\right) \leq c_{r^{0}}(p)-1$. We may thus write

$$
\begin{aligned}
\sum c_{r}\left(p^{\prime}\right) & =c_{r^{0}}\left(p^{\prime}\right)+\sum_{r \in\left\{r^{1}, \ldots, r^{m}\right\}} c_{r}\left(p^{\prime}\right)+\sum_{r \in S \backslash\left\{r^{0}, r^{1}, \ldots, r^{m}\right\}} c_{r}\left(p^{\prime}\right) \\
& \leq c_{r^{0}}(p)-1+\sum_{r \in\left\{r^{1}, \ldots, r^{m}\right\}} c_{r}\left(p^{\prime}\right)+\sum_{r \in S \backslash\left\{r^{0}, r^{1}, \ldots, r^{m}\right\}} c_{r}\left(p^{\prime}\right) \\
& \leq c_{r^{0}}(p)-1+\sum_{r \in\left\{r^{1}, \ldots, r^{m}\right\}} c_{r}(p)+\sum_{r \in S \backslash\left\{r^{0}, r^{1}, \ldots, r^{m}\right\}} c_{r}\left(p^{\prime}\right) \\
& =c_{r^{0}}(p)-1+\sum_{r \in\left\{r^{1}, \ldots, r^{m}\right\}} c_{r}(p)+\sum_{r \in S \backslash\left\{r^{0}, r^{1}, \ldots, r^{m}\right\}} c_{r}(p) \\
& =-1+\sum c_{r}(p) .
\end{aligned}
$$


Lemma 11. Assume $\varphi$ is strategy-proof, welfare anonymous, constant sequence continuous, and strongly undominated in these properties. Let $k \in \mathbb{Z}_{+}$, and $R \in \mathcal{D}^{k+1 / 2}$. Assume also that, for each $R^{\prime} \in \mathcal{D}^{k}, \varphi\left(R^{\prime}\right) \in F^{*}\left(R^{\prime}\right)$. Then $\varphi(R) \in F^{*}(R)$.

Proof. Let $i \in N$. Since $R_{i}^{p} \in \mathcal{R}, p^{*}\left(R_{i}^{p}, R_{-i}\right)=p$, and, since $F^{*}$ is strategy-proof, for each $y \in F^{*}\left(\mathcal{R}, R_{-i}\right), F^{*}\left(R_{i}^{p}, R_{-i}\right) R_{i}^{p} y$. It follows that each $y \in F^{*}\left(\mathcal{R}, R_{-i}\right)$ is affordable at prices $p$.

By Lemma 10, there is $t \in S$ and a sequence $\left\{R_{i}^{n}\right\} \subseteq \mathcal{T}\left(R_{i}^{p}, \llbracket p, t \rrbracket\right)$ such that $R_{i}^{n} \rightarrow R_{i}^{p}$ and, for each $n \in \mathbb{N},\left(R_{i}^{n}, R_{-i}\right) \in \mathcal{D}^{k}$. By hypothesis, for each $n \in \mathbb{N}, \varphi\left(R_{i}^{n}, R_{-i}\right) \in F^{*}\left(R_{i}^{n}, R_{-i}\right)$. The sequence of lower-contour sets $\left\{R_{i}^{n}\left[F^{*}\left(R_{i}^{n}, R_{-i}\right)\right]\right\}^{n \in \mathbb{N}}$ converges to the budget set $B:=\left\{\left(x_{i}, s\right) \in \mathbb{R} \times S: p_{s} x_{i} \leq 1\right\}$. By strategy-proofness, for each $n \in \mathbb{N}, \varphi_{i}(R) \in R_{i}^{n}\left[\varphi_{i}\left(R_{i}^{n}, R_{-i}\right)\right]=R_{i}^{n}\left[F_{i}^{*}\left(R_{i}^{n}, R_{-i}\right)\right]$. Conclude that $\varphi_{i}(R) \in B$.

Since $i \in N$ was arbitrary, in fact each bundle assigned at $\varphi(R)$ is in $B$. Strong undomination then yields $\varphi(R) \in F^{*}(R)$.

Lemma 12. Fix $k \in \mathbb{Z}_{+}$. Assume that, for each $R^{\prime} \in \mathcal{D}^{k-1 / 2}, \varphi\left(R^{\prime}\right) \in F^{*}\left(R^{\prime}\right)$. Let $R \in \mathcal{D}^{k}$. There is a sequence $\left\{R^{n}\right\} \subseteq \mathcal{D}^{k-1 / 2}$ such that $R^{n} \rightarrow R$; if $\varphi$ is constant sequence continuous, then $\varphi(R) \in F^{*}(R)$.

Proof. Let $R \in \mathcal{D}^{k} \backslash \mathcal{D}^{k-1 / 2}$ and $\alpha \in \mathcal{A}^{*}(R)$. Assume $\alpha$ is balanced. Let $s \in S$. By Lemma 5, there is a path $r \rightsquigarrow s$ in $\Gamma\left(R ; p^{*}(R), \alpha\right)$ such that $r$ is exhausted at $\llbracket p^{*}(R), \alpha \rrbracket$. Moreover, we may choose $r \rightsquigarrow s$ such that $r$ is the only exhausted site in the path. If there is $i \in N$ and $t \notin r \rightsquigarrow s$ such that i) $t$ is not exhausted at $\llbracket p^{*}(R), \alpha \rrbracket$ and ii) $(s, t, i) \in \Gamma\left(R ; p^{*}(R), \alpha\right)$, then append the arc $(s, t, i)$ to the path. Continue on in this way until it is no longer possible. Furthermore, we may repeat the process until each $\tilde{s} \in S$ is connected to one and only one exhausted site via our chosen paths (some paths may consist of a single, exhausted site and no arcs). Let $\Gamma^{\prime}$ be the graph resulting from the union of these paths. Clearly, $\Gamma^{\prime}$ is a minimal simple forest such that for each unexhausted $s \in S$, there is a path $r^{0} \leadsto s \subseteq \Gamma^{\prime}$ with $r^{0}$ exhausted.

For each $\varepsilon>0$, construct economy $R^{\varepsilon} \in \mathcal{R}^{N}$ as follows. For each $i \in N$, identify the set $\Gamma_{i}^{\prime}:=\left\{\tilde{s} \in S: \exists t \in S\right.$, s.t. $(\tilde{s}, t, i) \in \Gamma^{\prime}$ or $\left.(t, \tilde{s}, i) \in \Gamma^{\prime}\right\}$. Let $A_{i}:=\left\{\llbracket p, s \rrbracket \in \mathbb{R} \times S: s \in \Gamma_{i}^{\prime}\right\}$. Now let $R_{i}^{\varepsilon} \in \mathcal{T}\left(R_{i}, A_{i}\right)$ be such that $H^{\Delta}\left(R_{i}^{\varepsilon}, R_{i}\right)<\varepsilon$ (again, richness ensures $R_{i}^{\varepsilon} \in \mathcal{R}$ ). By construction, $\Gamma\left(R^{\varepsilon} ; p^{*}(R) \alpha\right)=\Gamma^{\prime}$. Moreover, since $\alpha$ is balanced, Theorem 2 implies $\llbracket p^{*}(R), \alpha \rrbracket \in F^{*}\left(R^{\varepsilon}\right)$. Therefore, $R^{\varepsilon} \in \mathcal{D}^{k-1 / 2}$. 
It is also easy to verify that for each $R^{\varepsilon},\left|F^{*}\left(R^{\varepsilon}\right)\right|=1$, and, moreover, for each pair $\varepsilon, \varepsilon^{\prime}$, $F^{*}\left(R^{\varepsilon}\right)=F^{*}\left(R^{\varepsilon^{\prime}}\right)$. Therefore, $\varphi\left(R^{\varepsilon}\right)=\varphi\left(R^{\varepsilon^{\prime}}\right)$. We generate the desired sequence $R^{n}$ via a decreasing sequence $\varepsilon^{n} \in \mathbb{R}$ such that $\varepsilon^{n} \rightarrow 0$. Constant sequence continuity then implies that $\varphi(R)$ is welfare equivalent to $F^{*}(R)$.

Now suppose there is $i \in N$ consuming above the $F^{*}(R)$ budget set: $\varphi_{i}(R)=\left(x_{i}, s\right)$ and $x>$ $p_{s}^{*}(R)^{-1}$. Instead have agent $i$ declare a sequence of preferences $R_{i}^{\varepsilon^{n}} \in \mathcal{T}\left(R_{i}^{p^{*}(R)}, A_{i}\right)$. The above arguments all go through: $\varphi\left(R_{i}^{p^{*}(R)}, R_{-i}\right)$ is welfare equivalent to $F^{*}\left(R_{i}^{p^{*}(R)}, R_{-i}\right)$, and, moreover, $p^{*}\left(R_{i}^{p^{*}(R)}, R_{-i}\right)=p^{*}(R)$. Thus at economy $\left(R_{i}^{p^{*}(R)}, R_{-i}\right), i$ prefers to report $R_{i}$, contradicting strategyproofness.

Proof of Theorem 5. Let $R \in \mathcal{R}^{N}$. There is $k \in \mathbb{Z}_{+}$such that $R \in \mathcal{D}^{k}$. Let $R^{k}:=R$. Let $\sigma^{\mathrm{XII}}\left(R^{k}\right) \subseteq \mathcal{D}^{k-1 / 2}$ be the sequence in Lemma 12. For each $R^{k-1 / 2} \in \sigma\left(R^{k}\right)$, there is a sequence $\sigma^{\mathrm{X}}\left(R^{k-1 / 2}\right) \subseteq \mathcal{D}^{k-1}$ as defined in Lemma 10. Collect these sequences in $\Sigma^{\mathrm{X}}(k-1)$. For each $\sigma^{\mathrm{X}}\left(R^{k-1 / 2}\right) \in \Sigma^{\mathrm{X}}(k-1)$, and each $R^{k-1} \in \sigma^{\mathrm{X}}\left(R^{k-1 / 2}\right)$, let $\sigma^{\mathrm{XII}}\left(R^{k-1}\right) \subseteq \mathcal{D}^{k-1-1 / 2}$ be the sequence in Lemma 12. Collect these sequences in $\Sigma^{\mathrm{XII}}(k-1-1 / 2)$. Continuing by recursion, we define the families $\left\{\Sigma^{\mathrm{X}}(k-q): q \in\{1, \ldots, k\}\right\}$ and $\left\{\Sigma^{\mathrm{XII}}(k-q-1 / 2): q \in\{1, \ldots, k\}\right\}$.

Fix $k^{\prime}-1 \in \mathbb{Z}_{+}$and $R \in \mathcal{D}^{k^{\prime}}$. Assume that for each $\sigma^{\mathrm{X}}\left(R^{k^{\prime}-1 / 2}\right) \in \Sigma^{\mathrm{X}}\left(k^{\prime}-1\right)$, and each $R \in$ $\sigma^{\mathrm{X}}\left(R^{k^{\prime}-1 / 2}\right), \varphi(R) \in F^{*}(R)$ (note that the base case, $k^{\prime}-1=0$, is proven by Proposition 3 ). Let $\sigma^{\mathrm{XII}}\left(R^{k^{\prime}}\right) \in \Sigma^{\mathrm{XII}}\left(k^{\prime}-1 / 2\right)$. Each $R^{k^{\prime}-1 / 2} \in \sigma^{\mathrm{XII}}\left(R^{k^{\prime}}\right)$ has an associated sequence $\sigma^{\mathrm{X}}\left(R^{k^{\prime}-1 / 2}\right) \in$ $\Sigma^{\mathrm{X}}\left(k^{\prime}-1\right)$, which satisfies the induction hypothesis. Since $\sigma^{\mathrm{X}}\left(R^{k^{\prime}-1 / 2}\right)$ is the sequence for $R^{k^{\prime}-1 / 2}$ constructed in Lemma 10, Lemma 11 then implies that $\varphi\left(R^{k^{\prime}-1 / 2}\right) \in F^{*}\left(R^{k^{\prime}-1 / 2}\right)$.

The previous implies that each $\tilde{R}^{k^{\prime}-1 / 2} \in \sigma^{\mathrm{XII}}\left(R^{k^{\prime}}\right)$ satisfies $\varphi\left(\tilde{R}^{k^{\prime}-1 / 2}\right) \in F^{*}\left(\tilde{R}^{k^{\prime}-1 / 2}\right)$. Since $\sigma^{\mathrm{XII}}\left(R^{k^{\prime}}\right)$ is the sequence constructed in Lemma 12 , the Lemma implies $\varphi\left(R^{k^{\prime}}\right) \in F^{*}\left(R^{k^{\prime}}\right)$.

\section{ApPendix C. Matroids, in BRIEF}

Our results depend on elementary matroid structure and one non-elementary result, the Edmonds Matroid Partition Theorem, which is itself a generalization of Hall's Marriage Theorem. We define, here, the structures necessary and state the theorem. For a less brief but nonetheless compact treatment, I recommend the chapter in Vohra (2004).

A matroid $\mathcal{M}$ consists of a finite ground set $N$ and a family $\mathcal{I} \subseteq 2^{N}$ with the following properties: (i) $\mathcal{I} \neq \emptyset$, (ii) if $A \in \mathcal{I}$ and $B \subseteq A$ then $B \in \mathcal{I}$, and (iii) if $\{A, B\} \subseteq \mathcal{I}$, and $|A|>|B|$ then there is $a \in A \backslash B$ such that $\{a\} \cup B \in \mathcal{I}$. Note that properties i-iii are also satisfied by the sets of linearly 
independent vectors in a finite-dimensional vector space, and by the paths in a graph. In fact, the elements of $\mathcal{I}$ are termed independent sets.

Alternatively, one often sees matroids defined as having properties i, ii, and the following: (iii') if $\{A, B\} \subseteq \mathcal{I}$, and both sets are maximal elements of $\mathcal{I}$ in the containment order, then $|A|=|B|$. We leave it to the reader to verify that, given $\mathrm{i}$ and ii, iii and iii' are equivalent.

Define the rank of a set $A \subseteq N$ as the cardinality of the cardinally-largest independent set contained in $A$. Thus, by property iii', the structure of a matroid can be captured by its rank function $\rho: 2^{\Omega} \rightarrow \mathbb{Z}$.

Let $\left(\mathcal{M}_{s}\right)_{s \in S}=\left(\left(N, \mathcal{I}_{s}\right)\right)_{s \in S}$ be a list of matroids all having the same ground set $N$. Let $\rho_{s}$ be the rank function of matroid $\mathcal{M}_{s}$. We say the matroids are partitionable, if there is a partition $\left(I_{s}\right)_{s \in S}$ of $N$ such that, for each $s \in S, I_{s} \in \mathcal{I}_{s}$. The following theorem is proved in Edmonds (1965).

Theorem (Edmonds Matroid Partition Theorem). A list of matroids $\left(\mathcal{M}_{s}\right)_{s \in S}$, with associated rank functions $\left(\rho_{s}\right)_{s \in S}$ is partitionable if and only if, for each subset $N^{\prime} \subseteq N,\left|N^{\prime}\right| \leq \sum_{s \in S} \rho_{s}\left(N^{\prime}\right)$.

\section{REFERENCES}

Ackermann, H., Goldberg, P. W., Mirrokni, V. S., Röglin, H., and Vöcking, B. (2007). A unified approach to congestion games and two-sided markets. In Deng, X. and Graham, F. C., editors, Internet and Network Economics, number 4858 in Lecture Notes in Computer Science, pages 30-41. Springer Berlin Heidelberg.

Alkan, A., Demange, G., and Gale, D. (1991). Fair allocation of indivisible goods and criteria of justice. Econometrica, 59(4):1023-1039.

Andersson, T. and Svensson, L.-G. (2014). Non-manipulable house allocation with rent control. Econometrica, 82(2):507-539.

Demange, G. and Gale, D. (1985). The strategy structure of two-sided matching markets. Econometrica, 53(4):873-888.

Edmonds, J. (1965). Minimum partition of a matroid into independent subsets. Journal of Research of the National Bureau of Standards Section B Mathematics and Mathematical Physics, 69B(1 and 2):67.

Gensemer, S. H., Hong, L., and Kelly, J. S. (1998). Migration disequilibrium and specific division rules. Social Choice and Welfare, 15(2):201-209. 
Gourvès, L., Monnot, J., Moretti, S., and Thang, N. K. (2012). Congestion games with capacitated resources. In Serna, M., editor, Algorithmic Game Theory, Lecture Notes in Computer Science, pages 204-215. Springer Berlin Heidelberg.

Hardin, G. (1968). The tragedy of the commons. Science, 162(3859):1243-1248.

Hildenbrand, W. (1974). Core and Equilibria of a Large Economy. Princeton university press Princeton.

Holmström, B. R. (1977). On Incentives and Control in Organizations. Stanford University.

Klaus, B. and Bochet, O. (2013). The relation between monotonicity and strategy-proofness. Social Choice and Welfare, 40(1):41-63.

Leonard, H. B. (1983). Elicitation of honest preferences for the assignment of individuals to positions. Journal of Political Economy, 91(3):461-479.

Miyagawa, E. (2001). House Allocation with Transfers. Journal of Economic Theory, 100(2):329_ 355.

Monderer, D. and Shapley, L. S. (1996). Potential games. Games and Economic Behavior, 14(1):124-143.

Morimoto, S. and Serizawa, S. (2015). Strategy-proofness and efficiency with non-quasi-linear preferences: A characterization of minimum price Walrasian rule. Theoretical Economics, 10(2):445-487.

Schummer, J. (2000). Eliciting preferences to assign positions and compensation. Games and Economic Behavior, 30(2):293-318.

Shapley, L. S. and Shubik, M. (1971). The assignment game I: The core. International Journal of Game Theory, 1(1):111-130.

Svensson, L.-G. (2009). Coalitional strategy-proofness and fairness. Economic Theory, 40(2):227245.

Svensson, L.-G. and Larsson, B. (2002). Strategy-proof and nonbossy allocation of indivisible goods and money. Economic Theory, 20(3):483-502.

Thomson, W. (2014). Strategy-proof allocation rules. Unpublished manuscript.

Tierney, R. (2016). Strategy-proof assignment of objects and money. Unpublished manuscript.

U.S. General Accounting Office (2002). Individual fishing quotas: Better information could improve program management. http://www.gao.gov/products/GAO-03-159.

Vohra, R. V. (2004). Advanced Mathematical Economics. Routledge. 